\title{
Resveratrol Alleviates Skeletal Muscle Insulin Resistance by Downregulating Long Noncoding RNA
}

\author{
Zhihong Liu $\mathbb{D}^{1},{ }^{1,2,3}$ Zhimei Zhang, ${ }^{1,2}$ Guangyao Song $\mathbb{D}^{1,2}$ Xing Wang $\mathbb{D}^{4},{ }^{4}$ Hanying Xing $\mathbb{D}^{4}{ }^{4}$ \\ and Chao Wang iD ${ }^{4}$ \\ ${ }^{1}$ Department of Internal Medicine, Hebei Medical University, Shijiazhuang 050017, China \\ ${ }^{2}$ Endocrinology Department, Hebei General Hospital, Shijiazhuang 050051, China \\ ${ }^{3}$ Endocrinology Department, The Second Hospital of Hebei Medical University, Shijiazhuang 050000, China \\ ${ }^{4}$ Hebei Key Laboratory of Metabolic Diseases, Hebei General Hospital, Shijiazhuang 050051, China
}

Correspondence should be addressed to Guangyao Song; sguangyao2@163.com

Received 6 July 2021; Revised 15 December 2021; Accepted 4 January 2022; Published 19 January 2022

Academic Editor: Rosaria Meccariello

Copyright ( $\odot 2022$ Zhihong Liu et al. This is an open access article distributed under the Creative Commons Attribution License, which permits unrestricted use, distribution, and reproduction in any medium, provided the original work is properly cited.

Long noncoding RNA (lncRNA) is a crucial factor in the progression of insulin resistance (IR). Resveratrol (RSV) exhibits promising therapeutic potential for IR. However, there are few studies on whether RSV improves IR through lncRNA. This study aimed to determine whether RSV could influence the expression of lncRNA and to elucidate the underlying mechanism. Mice were divided into three groups: control group, high-fat diet (HFD) group, and HFD + RSV group. We conducted a highthroughput sequencing analysis to detect lncRNA and mRNA expression signatures and the ceRNA-network in the skeletal muscles of mice that were fed an HFD to induce IR. Hierarchical clustering, gene enrichment, and gene ceRNA-network analyses were subsequently conducted. Differentially expressed lncRNAs were selected and validated via reverse transcription-quantitative polymerase chain reaction (RT-qPCR). The biological functions of the selected lncRNAs were investigated by silencing the target genes via lentivirus transfection of $\mathrm{C} 2 \mathrm{C} 12$ mouse myotube cells. RSV treatment reversed the expression of 338 mRNAs and 629 lncRNAs in the skeletal muscles of mice with HFD-induced IR. The results of the Gene Ontology and Kyoto Encyclopedia of Genes and Genomes database analyses indicated that the differentially expressed mRNAs modulated type II diabetes mellitus. After validating randomly selected lncRNAs via RT-qPCR, we identified a novel lncRNA, NONMMUT044897.2, which was upregulated in the HFD group and reversed with RSV treatment. Additionally, NONMMUT044897.2 was proven to function as a ceRNA of microRNA- (miR-) 7051-5p. Suppressor of Cytokine Signaling 1 (SOCS1) was confirmed as a target of miR-7051-5p. We further performed lentivirus transfection to knock down NONMMUT044897.2 in vitro and found that NONMMUT044897.2 silenced SOCS1 and potentiated the insulin signaling pathway. Hence, RSV mimicked the silencing effect of lentivirus transfection on NONMMUT044897.2. Our study revealed that RSV reduced IR in mouse skeletal muscles via the regulation of NONMMUT044897.2.

\section{Introduction}

Type 2 diabetes mellitus (T2DM) accounts for $90 \%$ of diabetes worldwide, and insulin resistance (IR) is a primary determinant of T2DM since it reduces glucose uptake and utilization [1]. Skeletal muscles play a significant role in the etiology of IR [2]. Phosphatidylinositol-3-kinase (PI3K)/ protein kinase B (AKT) is the most important signaling pathway of insulin in glucose metabolism [3]. Activated AKT can promote phosphorylated GSK $3 \beta$, inhibiting its activity to improve IR [4]. In recent years, researchers found that the Suppressor of Cytokine Signaling 1 (SOCS1) is an important negative regulator of the PI3K/AKT pathway $[5,6]$. Overexpression of SOCS1 in the liver was found to reduce insulin sensitivity by downregulating the level of tyrosine phosphorylation of insulin receptor substrate in IR mice [5]. SOCS1 primarily inhibits the catalytic binding of insulin receptors to insulin receptor substrates to induce IR [6].

Resveratrol (3,5,4-trihydroxystilbene; RSV) is a natural polyphenol that is enriched in more than 70 kinds of plants 
[7]. Accumulating evidence has indicated that RSV has diverse biological activities $[8,9]$. It functions as an antioxidant, antiaging, anti-inflammatory, hypoglycemic, and anti-IR agent $[10,11]$. Several studies have reported that RSV possesses a significant anti-IR activity in skeletal muscles [12-14]. Our earlier research has also proven that skeletal muscle IR that is caused by an HFD may be alleviated with RSV treatment [14]. RSV increases the expression of microencapsulated protein $3(\mathrm{CAV}-3)$, thereby allowing skeletal muscle cells to carry glucose when the protein GLUT4 activates the transfer from the cytoplasm to the cell membrane, which in turn increases the ability of myocytes to transport glucose and improve IR [15]. Meanwhile, RSV attenuates insulin-stimulated AKT phosphorylation by eliminating insulin-induced ROS production in skeletal muscles [16].

Long noncoding RNAs (lncRNAs), a class of RNA molecules that are more than 200 nucleotides (nt) in length, have little or no protein-coding capacity [17]. Research has shown that $\operatorname{lncRNAs}$ widely participate in various developmental and physiological processes $[18,19]$. In addition, they are strongly correlated with the development and progression of diseases, including coronary artery diseases [20], cancers [21], and metabolic diseases [22, 23]. Recently, lncRNAs have been confirmed to inhibit miRNA activity, increase miRNA target genes, and act as competitive endogenous RNAs (ceRNA) of miRNAs [24]. Further, the effect of lncRNAs in IR has been the focus of several studies $[25,26]$. Previous studies $[27,28]$ by our group have demonstrated that RSV can improve hepatic IR by regulating lncRNA NONMMUT058999.2 and NONMMUT008655.2 in mouse models. However, the effect of RSV on IR through the regulation of the expression of lncRNAs in skeletal muscle remains unclear. In this study, we aimed to establish in vivo and in vitro IR models in skeletal muscles, which are a different tissue than those used in previous studies, to explore whether RSV can improve skeletal muscle IR by regulating lncRNAs.

\section{Materials and Methods}

2.1. Animal Experiments. We equally divided 42 healthy 6week-old C57BL/6J background mice into three groups ( $n=14$ in each group): the control group, the HFD group, and the HFD + RSV group. The mice weighing around $22 \mathrm{~g}$ were purchased from the Beijing Viton Lihua Experimental Center (China) and sustained on a standard $12 \mathrm{~h}$ light-dark cycle, at $20-25^{\circ} \mathrm{C}$ and at $40-60 \%$ humidity. HFD mice were fed D12492J (20\% protein, $20 \%$ carbohydrate, and 60\% fat) for 8 weeks. The feed was purchased from Beijing Huafukang Biotechnology Co., Ltd. HFD + RSV mice were intragastrically fed $100 \mathrm{mg} / \mathrm{kg} /$ day RSV solution for 6 weeks following the methods outlined in our previous study [27]. Dissolved RSV (Sigma Aldrich, St. Louis, MO, USA) with dimethyl sulfolane (Sigma Aldrich; $30 \mathrm{mg} \mathrm{mL}^{-1}$ ) was diluted with $0.9 \% \mathrm{NaCl}$ in a ratio of $1: 2$. The control group was fed D12450J (20\% protein, 70\% carbohydrate, and 10\% fat). Weight and food intake were measured weekly during feeding. After the feeding experiment, all mice fasting for
$12 \mathrm{~h}$ were intraperitoneally injected with $50 \%$ glucose (1.5 $\mathrm{g} \mathrm{kg}^{-1}$ bodyweight) to conduct the glucose tolerance tests (intraperitoneal glucose tolerance test). Blood glucose was detected on the tail vein with a glucose meter at $0,15,30$, 60 , and $120 \mathrm{~min}$ after injection. The IR model was validated by determining the area under the curve in accordance with the protocol presented in our previously published study [29]. Animal studies (2019E369) were approved by the ethics committee of the Hebei General Hospital, and all animal experimental procedures complied with the National Institutes of Health guide for the care and use of laboratory animals.

2.2. Serum and Tissue Samples. Six mice in each group were randomly selected and intraperitoneally injected with $1.5 \mathrm{U} /$ $40 \mathrm{~g}$ of insulin (Sigma Aldrich). All mice in three groups were anesthetized by intraperitoneal injection of $2 \%$ sodium pentobarbital after $20 \mathrm{~min}$. Blood samples were gathered via cardiac puncture and centrifuged at $3000 \times g$ at $4^{\circ} \mathrm{C}$ for $10 \mathrm{~min}$. The serum was then stored at $-80^{\circ} \mathrm{C}$ for serological indicators. Collected skeletal muscles were withdrawn quickly and stored in liquid nitrogen for the follow-up study.

2.3. Serological Indicators. Detection kits for total cholesterol, triglyceride, high-density lipoprotein cholesterol, lowdensity lipoprotein cholesterol, and free fatty acids were acquired from Nanjing Jiancheng Institute of Biological Engineering (Jiangsu, China). Serum insulin was obtained using an ELISA kit (ALPCO Diagnostics, Salem, NH, USA). The manufacturer's protocol was followed in all the aforementioned procedures involving the detection kits.

2.4. Western Blot. The same amount of protein with different groups was separated by sodium dodecyl sulfate-polyacrylamide gel electrophoresis, transferred to polyvinylidene fluoride (PVDF) membrane (cat. no. ISEQ00010; Merk Millipore, Billerica, MA, USA), and sealed with 5\% skimmed milk for $2 \mathrm{~h}$. The primary antibodies were diluted in the blocking solution at the following concentrations: $\beta$-actin (cat. no. 4970; Cell Signaling Technology, Danvers, MA, USA): rabbit antibody, 1:5000; GAPDH (cat. no. 10494-1AP; Proteintech Group, Inc. 5400 Pearl Street, Suite 300 Rosemont, IL 60018, USA): rabbit antibody, $1: 10000$; AKT (cat. no. 9272; Cell Signaling Technology): rabbit antibody, 1:1000; p-AKT (Ser 473) (cat. no. 9271; Cell Signaling Technology): rabbit antibody, $1: 750$; GSK3 $\beta$ (cat. no. 12456; Cell Signaling Technology): rabbit antibody, 1:750; p-GSK3 $\beta$ (cat. no. 5558; Cell Signaling Technology): rabbit antibody, 1:750; GLUT4 (cat. no. 2213; Cell Signaling Technology): mouse antibody, 1:5000; SOCS1 (cat. no. 3950; Cell Signaling Technology): rabbit antibody, 1:5000. PVDF membranes and primary antibodies were incubated at $4^{\circ} \mathrm{C}$ for $24 \mathrm{~h}$. The membrane was then treated with secondary antibodies, stored at $30^{\circ} \mathrm{C}$ for about $50 \mathrm{~min}$ and washed three times for $10 \mathrm{~min}$ each time. The washed membrane was fully immersed in the iPer ECL Western HRP Substrate (cat. no. MF074-01; Mei5, Beijing, China) for about $2 \mathrm{~min}$ and 
exposed using the Gel Imager System (GDS8000; UVP, California, USA) to obtain the image of the target band. Protein bands were calculated by densitometry using the ImageJ software and were normalized to $\beta$-actin or GAPDH levels.

\subsection{Reverse Transcription-Quantitative Polymerase Chain} Reaction (RT-qPCR). Total RNA was extracted using the Trizol reagent (TIANGEN, Beijing, China) from mouse skeletal muscle tissues and was reverse-transcribed into cDNA using HiScript II Q RT SuperMix for qPCR. RNA was tested for purity and concentration using NanoDrop 2000 (Thermo Fisher Scientific, Wilmington, DE, USA). Amplification was performed using the SYBr ${ }^{\circledR}$ Premix ex Taq II kit (RR820A; Takara Bio, Tokyo, Japan). The Applied Biosystems 7500 real-time PCR system was used to perform RTqPCR, with a total of 41 cycles, including 3 minutes of predenaturation at $95^{\circ} \mathrm{C}, 5$ seconds at $95^{\circ} \mathrm{C}$, and 32 seconds at $60^{\circ} \mathrm{C}$. The melting point curve was established at $60-95^{\circ} \mathrm{C}$. $\beta$-Actin and U6 were considered as internal reference controls for genes. The relative gene expression was quantified by the $2^{-(\Delta \Delta \mathrm{Ct})}$ method [30]. The specific primers involved in this research are listed in Table 1.

2.6. cDNA Library Construction and RNA Sequencing. Total RNA was extracted using the RNeasy mini kit (Qiagen, Hilden, Germany) from skeletal muscle samples of four mice in each group. The TruSeq ${ }^{\mathrm{TM}}$ RNA Sample Preparation Kit (Illumina, San Diego, CA, USA) was used to make pairedend libraries following the manufacturer's instructions. Ribosomal RNA was removed using the Ribo-Zero rRNA Removal Kit (Epicentre, Madison, Wisconsin, USA), and then the mRNA was fragmented into small pieces with divalent cations. First chain cDNA was synthesized using reverse transcriptase and random primers, and second chain cDNA was generated by DNA Polymerase I and RNase $H$. Base "A" was added at the end of the cDNAs. Purified products were PCR-amplified to create the final cDNA library. Insert sizes were confirmed using a Qubit ${ }^{\circledR} 2.0$ Fluorometer (Life Technologies, Carlsbad, CA, USA), and mole concentrations were calculated. Clusters were created using cBot and then sequenced on the Illumina NovaSeq 6000 (Illumina) by Sinotech Genomics Co., Ltd. (Shanghai, China). Each gene fragment was counted using the Stringtie software (version: 1.3.0; Johns Hopkins University, Baltimore, MD, USA) contrast and then normalized using the TMM (trimmed mean of $M$ values) algorithm. The Fragments Per Kilobase Million value of each gene was then calculated. The high-throughput sequencing results were uploaded to the gene expression omnibus database (accession no. GSE178415).

\section{Analysis of Differentially Expressed lncRNA and mRNA}

The differential expression of skeletal muscle in the three groups was analyzed based on the "edge" package in R. The threshold of the $P$-value was confirmed by controlling the
False Discovery Rate. The screening criteria for differential expression of mRNAs and lncRNAs were $P<0.05$ and absolute value of $\log 2 \mathrm{FC}>1.0$.

3.1. Functional Group Analysis. The Gene Ontology (GO) and the Kyoto Encyclopedia of Genes and Genomes (KEGG) pathways determined the potential role of the lncRNAs that were coexpressed with the differentially expressed mRNAs. The GO analysis was conducted to establish significant annotations of genes and gene products in diversified organisms using the DAVID database (https://david.abcc. ncifcrf.gov). In addition, the KEGG pathway analysis was used to identify differentially expressed mRNAs in enriched pathways. $P<0.05$ was identified as the threshold of significance.

3.2. Construction of a ceRNA Regulatory Network. Cytoscape (version 3.8.2) is a network visualization software with multiple applications for network analysis. It can be downloaded for free from https://www.cytoscape.org/.

3.3. $\mathrm{C} 2 \mathrm{C} 12$ Cell Culture and Treatments. $\mathrm{C} 2 \mathrm{C} 12$ mouse myotube cells were maintained in Dulbecco's modified Eagle medium (Gibco, Waltham, MA, USA) at a density of $5 \times 10^{4}$ cells $/ \mathrm{cm}^{2}$ containing $10 \%$ fetal bovine serum (San Diego, California, USA) and $1 \%$ penicillin/streptomycin (Wisent, Nanjing, China) at $37^{\circ} \mathrm{C}$ with $5 \% \mathrm{CO}_{2}$. Cell differentiation was induced by incubation in Dulbecco's modified Eagle medium containing $2 \%$ fetal bovine serum for 4 days after reaching $80 \%$ confluence. Differentiated C2C12 cells at a density of $1 \times 10^{4}$ cells $/ \mathrm{cm}^{2}$ were incubated for $24 \mathrm{~h}$ with $0.25 \mathrm{mM}$ palmitate (PA) (Aladdin Industries, China) [31]. At $0,8,16$, and $24 \mathrm{~h}$ after the intervention of PA, the glucose concentration in the culture medium was measured by the glucose oxidase assay to determine whether IR in mice was established. Subcultured C2C12 cells were digested to prepare a cell suspension and subcultured to a 96-well culture plate. When the cells grew to about $80 \%$ confluence, RSV at concentrations of $100 \mu \mathrm{M}, 50 \mu \mathrm{M}$, and $30 \mu \mathrm{M}$ was added to the medium. After $24 \mathrm{~h}, 10 \mu \mathrm{L}$ of CCK- 8 was added to each well, which was protected against light. They were cultured for $20 \mathrm{~min}$, and their absorbance was measured at $450 \mathrm{~nm}$. The cell survival rate was then calculated. $\mathrm{C} 2 \mathrm{C} 12$ cells $\left(5 \times 10^{5} \mathrm{cells} / \mathrm{cm}^{2}\right)$ in the logarithmic growth phase were subcultured in a 6-well plate and transfected with lentivirus. Synthesized constructs included LV3-NC ( $5^{\prime}$ to $3^{\prime}$ TTCT CCGAACGTGTCACGT) and LV3-NONMMUT044897.2 ( $5^{\prime}$ to $3^{\prime}$ GCTCTTTCAGATAAGCCTTGT), which were obtained from GenePharma Co., Ltd. China. Stable cell lines were obtained after puromycin $\left(2 \mu \mathrm{g} \mathrm{mL}^{-1}\right)$ selection for the PA-induced IR model and drug intervention experiments. The plated cells were grouped: the control group $(\mathrm{CON})$, the PA group (PA), the PA + shRNA-NONMMUT044897.2 negative control group (PA+shRNA-NC), the PA + shRNA-NONMMUT044897.2 knockdown group (PA+shRNA-NONMMUT044897.2), and the PA+RSV $30 \mu \mathrm{M}$ group $(\mathrm{PA}+\mathrm{RSV})$. After the IR model was 


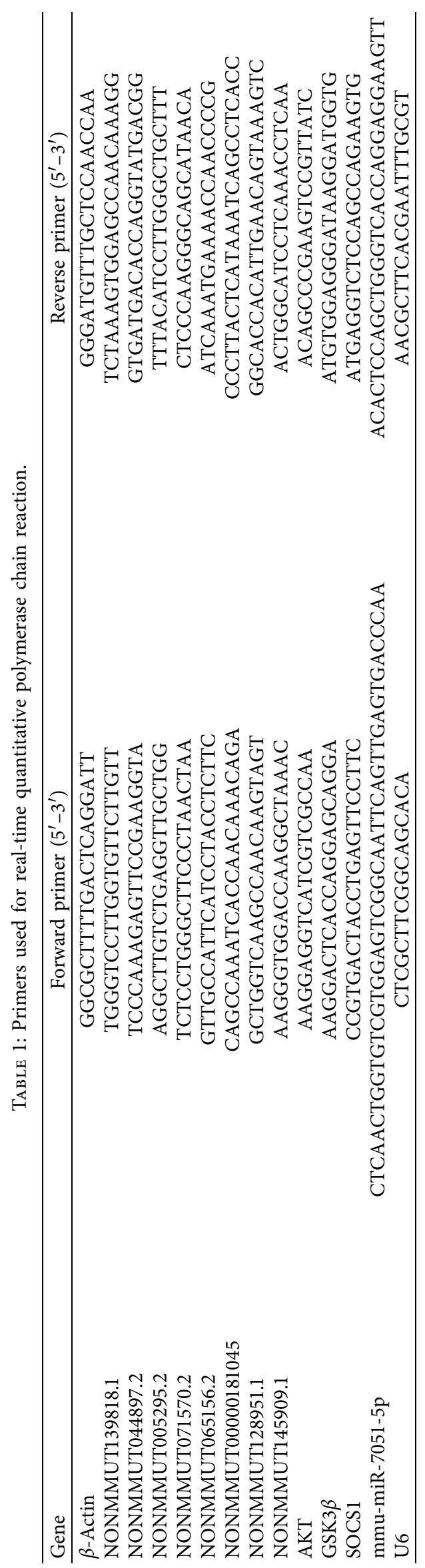


established, the glucose concentration was measured for $24 \mathrm{~h}$, while the NONMMUT044897.2 and miR7051-5p mRNA levels were measured via RT-qPCR. The cells at a density of $5 \times 10^{5}$ cells $/ \mathrm{cm}^{2}$ were stimulated with $100 \mathrm{nM}$ insulin, while the protein was extracted $20 \mathrm{~min}$ after insulin stimulation for the western blot analyses, and cells were extracted using the Trizol reagent (TIANGEN, Beijing, China).

3.4. Statistical Analyses. SPSS v23.0 was used for data analysis. The results are presented as a mean \pm SD. Twosample comparisons were analyzed using an independent sample $t$-test (Student's $t$-test). One-way ANOVA was used for statistical analysis followed by Bonferroni's multiple comparison test or Tamhane's multiple comparison test. $P<0.05$ was regarded as statistically significant.

\section{Results}

4.1. RSV Ameliorates Body Weight, IR, and Lipid Levels in HFD-Fed Mice. After 6 weeks of RSV administration, body weight, fasting blood glucose, and insulin levels of the $\mathrm{HFD}+\mathrm{RSV}$ group were greatly reduced compared with those of the HFD group (Table 2), although the daily caloric intake of the two groups was similar. The quantitative insulin sensitivity index of the HFD group was reduced compared with the indices of the CON and HFD + RSV groups (Table 2). RSV treatment reduced the upregulation of triglyceride, low-density lipoprotein cholesterol, and free fatty acids in the HFD group, while the total cholesterol was decreased but had no significance (Table 2). There was no difference in the high-density lipoprotein cholesterol of the mice (Table 2).

\subsection{RSV Treatment Decreased SOCS1 and Increased the} Phosphorylation of AKT and GSK3 $\beta$ in the HFD Group. Between the control, HFD, and HFD + RSV groups, no differences were found in the protein levels of AKT and GSK3 $\beta$ (Figures 1(a), 1(b), and 1(d)). The HFD group showed dramatic repression of $\mathrm{p}-\mathrm{AKT}$ and $\mathrm{p}-\mathrm{GSK} 3 \beta$ protein levels compared with those in the control group, while the RSV group showed a marked increase in p-AKT and p-GSK3 $\beta$ protein expression (Figures $1(\mathrm{a}), 1(\mathrm{c})$, and $1(\mathrm{e})$ ). Moreover, SOCS1 expression was abnormally elevated in the HFD group but decreased following RSV treatment (Figures 1(a) and 1(f)). These results suggest that RSV improves the expression of genes on the insulin signaling pathway.

4.3. RSV Systematically Modulates Skeletal Muscle Gene Expression. After standardization, 58,245 lncRNAs and 83,089 mRNAs were identified in the skeletal muscles of mice. On comparing the HFD group with the control group, we found that there were 3,276 differentially expressed lncRNAs (1,192 upregulated and 2,084 downregulated) and 2,118 differentially expressed mRNAs (314 upregulated and 1804 downregulated). Simultaneously, there were 1,640 differentially expressed lncRNAs (921 upregulated and 719 downregulated) and 604 differentially expressed mRNAs (444 upregulated and 160 downregulated) in the HFD + RSV group compared with those in the HFD group. Of the upregulated lncRNAs and mRNAs in the HFD group, 270 lncRNAs and 58 mRNAs were downregulated in the HFD + RSV group. Of the downregulated lncRNAs and mRNAs in the HFD group, 359 lncRNAs and 280 mRNAs were upregulated in the HFD + RSV group (Figures 2(a) and 2(b)). The top 30 differentially expressed lncRNAs and mRNAs are listed in Tables 3 and 4, with Fragments Per Kilobase Million $=0$ eliminated. All differentially expressed lncRNAs and mRNAs in the three groups are listed in Supplementary Tables S1 and S2.

4.4. Functional Enrichment Analysis of Differentially Expressed Genes. The functions of these different mRNAs were studied via enrichment analysis. The GO analysis was conducted to classify differentially expressed mRNAs into three types: the biological process, the molecular function, and the cellular component. The most highly enriched GO terms were "myelination, ensheathment of neurons, axon ensheathment, cellular component assembly involved in morphogenesis, transition between fast and slow fiber (biological process)," "myofibril, sarcomere, contractile fiber, compact myelin, contractile fiber part (cellular component)," and "structural constituent of myelin sheath, actin binding, fatty acid synthase activity, ion gated channel activity, and gated channel activity (molecular function)" (Figure 3(a)). KEGG analysis revealed that the differentially expressed mRNAs were mostly involved in cytokine-cytokine receptor interaction, the JAK-STAT signaling pathway, hypertrophic cardiomyopathy, the prolactin signaling pathway, and type II diabetes mellitus (Figure 3(b)).

4.5. RT-qPCR Validation In Vivo. To confirm the validity of the sequencing results, we randomly picked four differentially expressed lncRNAs, two upregulated lncRNAs in HFD, a downregulated HFD + RSV (NONMMUT044897.2; NONMMUT005295.2), and two lncRNAs with a reverse trend (NONMMUT128951.1; NONMMUT145909.1). The expression levels of the selected lncRNAs were consistent with those of the sequencing results (Figures 4(a)-4(d)), but there was no statistical difference in the increase of NONMMUT128951.1 in the HFD+RSV group. The SOCS1 mRNA level was increased in the HFD group and decreased with RSV treatment (Figure 4(f)). Of the verified lncRNAs, NONMMUT044897.2 had a higher expression level. In addition, the KEGG analysis indicated that SOCS1 played a vital role in the JAK-STAT signaling pathway and in type II diabetes mellitus. In the latter, SOCS1 was reportedly involved in the development of IR $[5,6]$. To elucidate the interaction between NONMMUT044897.2 and SOCS1, we constructed a related lncRNA-miRNA-mRNA network diagram. The results revealed that NONMMUT044897.2 regulated SOCS1 through miR-7051-5p and miR-762 (Figure 4(g)). To further explore its potential molecular mechanism, we applied the NonCode and miRBase database 
TABLE 2: General indicators after resveratrol treatment.

\begin{tabular}{|c|c|c|c|}
\hline Name & CON group $(n=14)$ & HFD group $(n=14)$ & HFD + RSV group $(n=14)$ \\
\hline Initial body weight (g) & $22.40 \pm 1.22$ & $22.95 \pm 1.11$ & $22.32 \pm 1.14$ \\
\hline Final body weight (g) & $27.04 \pm 2.56$ & $41.61 \pm 3.71^{*}$ & $38.83 \pm 2.27^{\#}$ \\
\hline Food intake $(\mathrm{kcal} / \mathrm{d})$ & $12.89 \pm 0.15$ & $12.50 \pm 0.30$ & $12.52 \pm 0.72$ \\
\hline $\mathrm{TC}(\mathrm{mmol} / \mathrm{L})$ & $6.06 \pm 0.72$ & $7.62 \pm 0.52^{*}$ & $7.44 \pm 0.43$ \\
\hline $\mathrm{TG}(\mathrm{mmol} / \mathrm{L})$ & $0.51 \pm 0.11$ & $1.42 \pm 0.22^{*}$ & $0.86 \pm 0.07^{\#}$ \\
\hline HDL-C (mmol/L) & $3.26 \pm 0.22$ & $3.01 \pm 0.30$ & $3.14 \pm 0.37$ \\
\hline $\mathrm{LDL}-\mathrm{C}(\mathrm{mmol} / \mathrm{L})$ & $0.21 \pm 0.02$ & $0.64 \pm 0.07^{*}$ & $0.47 \pm 0.04^{\#}$ \\
\hline FFA $(\mathrm{mmol} / \mathrm{L})$ & $0.73 \pm 0.08$ & $1.22 \pm 0.14^{*}$ & $0.93 \pm 0.05^{\#}$ \\
\hline $\mathrm{FBG}(\mathrm{mmol} / \mathrm{L})$ & $5.9 \pm 0.70$ & $12.42 \pm 1.96^{*}$ & $8.14 \pm 0.85^{\#}$ \\
\hline Insulin ( $\mathrm{ng} / \mathrm{mL})$ & $0.20 \pm 0.20$ & $1.35 \pm 0.28^{*}$ & $0.51 \pm 0.21^{\#}$ \\
\hline QUICKI & $0.83 \pm 0.17$ & $0.41 \pm 0.02 *$ & $0.55 \pm 0.05^{\#}$ \\
\hline
\end{tabular}

${ }^{*} P<0.05$ versus CON; ${ }^{\#} P<0.05$ versus HFD. TC: total cholesterol; TG: triglyceride; HDL-C: high-density lipoprotein cholesterol; LDL-C: low-density lipoprotein cholesterol; FFA: free fatty acid; FBG: fasting blood sugar; QUICKI: quantitative insulin sensitivity index; HFD: high-fat diet.

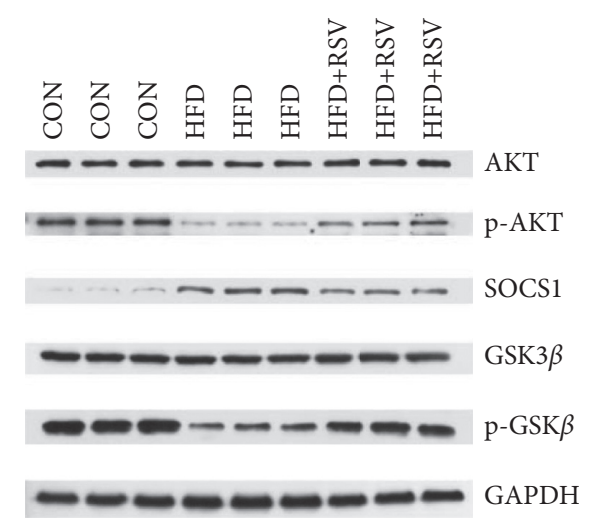

(a)

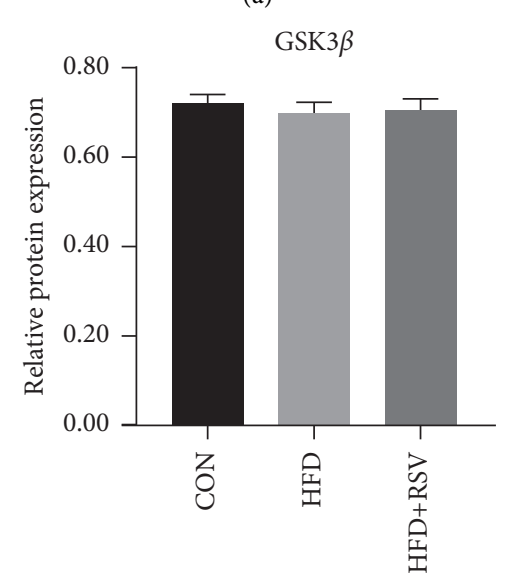

(d)

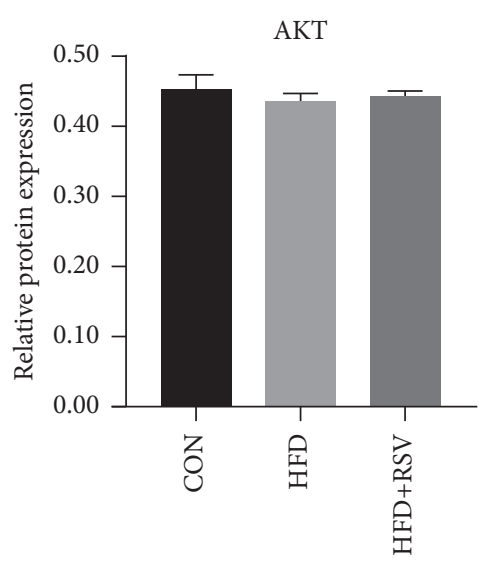

(b)

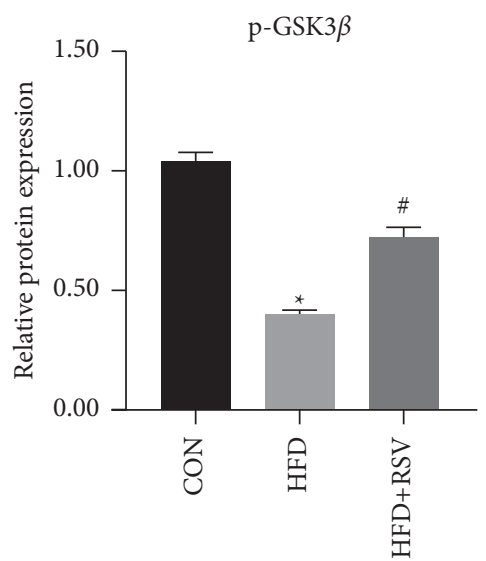

(e)

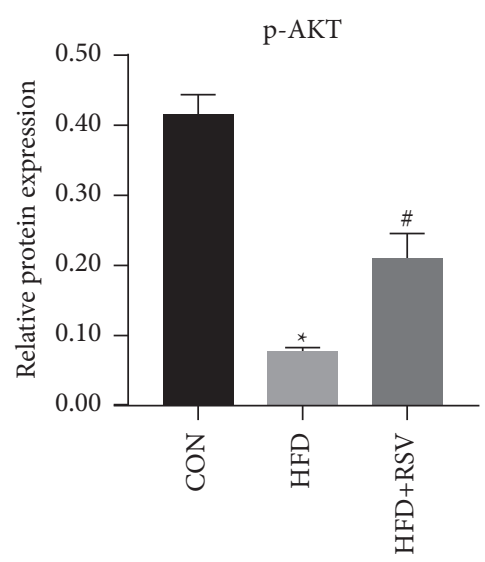

(c)

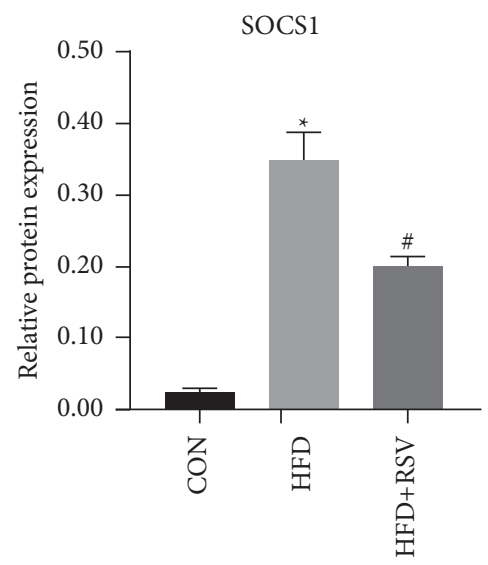

(f)

FIgURE 1: Effects of resveratrol on the insulin signaling pathway in control, HFD, and HFD + RSV groups. (a) Bands of western blot; (b) AKT; (c) p-AKT; (d) GSK3 $\beta$; (e) p-GSK3 $\beta$; (f) SOCS1. Data are expressed as the mean \pm SD $(n=6) .{ }^{*} P<0.05$ versus CON; ${ }^{\#} P<0.05$ versus HFD.

analyses and found that there was base pairing in the sequence of NONMMUT044897.2 and miR-7051-5p. At the same time, according to the prediction results of TargetScan, SOCS1 was identified to be a miR-7051-5p target (Figure 4(h)). According to the above results, NONMMUT044897.2 might have regulated SOCS1 through miR7051-5p. Therefore, this study further verified the expression of miR-7051-5p mRNA through RT-qPCR; miR-7051-5p mRNA was downregulated in the HFD group and upregulated in the HFD + RSV group (Figure 4(e)).

4.6. Establishment of a Cell Model of PA-Induced IR. The $\mathrm{C} 2 \mathrm{C} 12$ mouse myotube cells were transferred to media with 


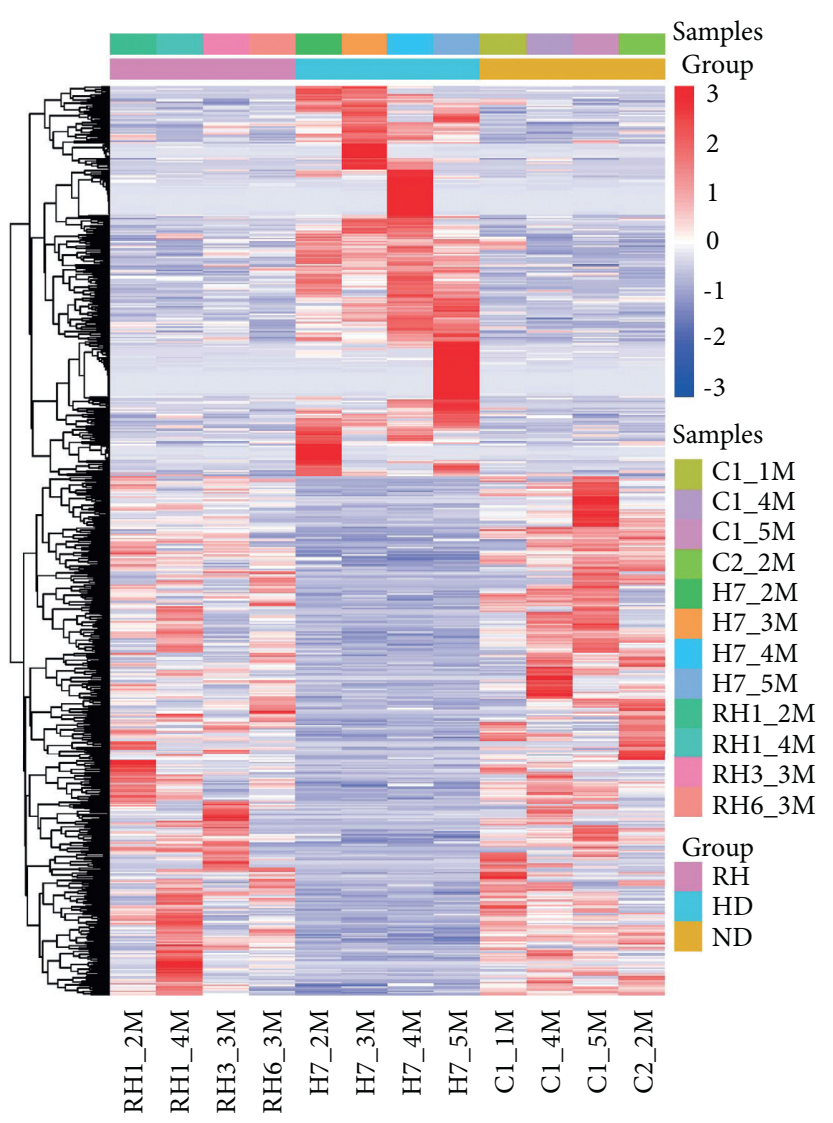

(a)

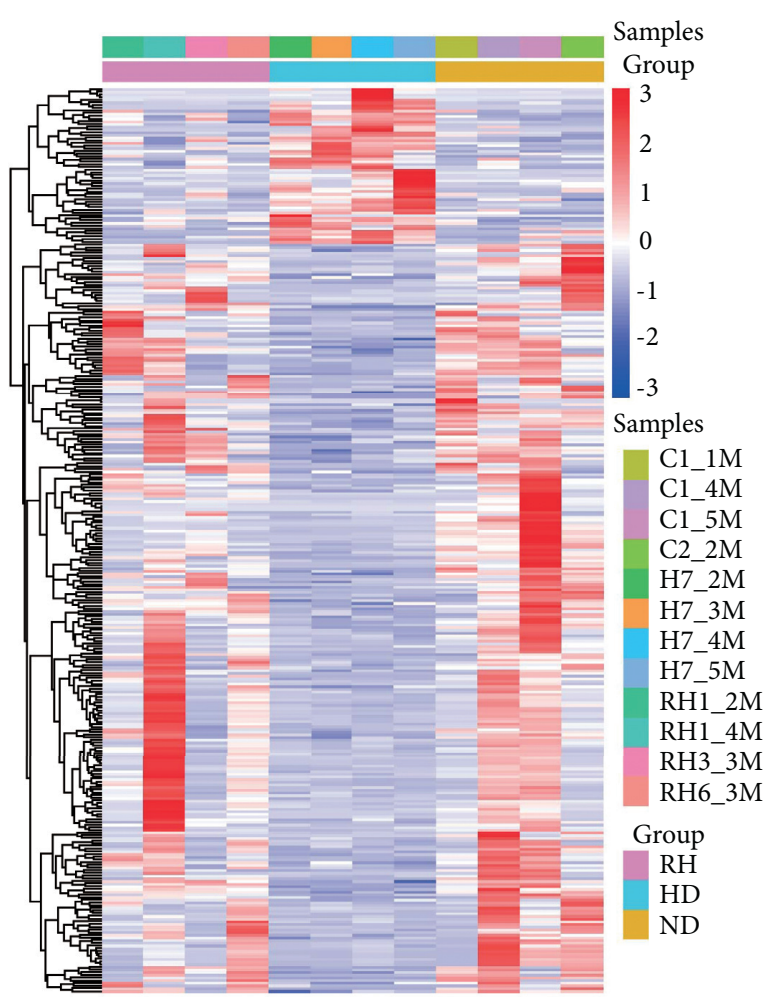

$\sum_{j} \sum_{m} \sum_{m} \sum_{j} \sum_{j} \sum_{i} \sum_{j} \sum_{i} \sum_{i}$

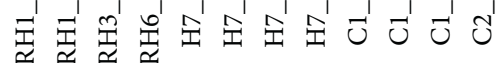

(b)

FIgURe 2: Profiles of differentially expressed genes in the three groups. Hierarchical clustering of lncRNAs (a) and mRNAs (b). Upregulated and downregulated lncRNAs (or mRNAs) are indicated in red and blue.

TABLe 3: Top 30 significantly differentially expressed lncRNAs in mice.

\begin{tabular}{|c|c|c|c|c|c|c|}
\hline LncRNA_id & $\begin{array}{l}\log 2 \mathrm{FC}(\mathrm{HFD} \\
\text { versus CON })\end{array}$ & $\begin{array}{l}P \text { value }(\mathrm{HFD} \\
\text { versus } \mathrm{CON})\end{array}$ & $\begin{array}{c}\text { Up/down } \\
\text { (HFD versus } \\
\text { CON) }\end{array}$ & $\begin{array}{c}\log 2 \mathrm{FC} \\
(\mathrm{HFD}+\mathrm{RSV} \\
\text { versus HFD }) \\
\end{array}$ & $\begin{array}{c}P \text { value } \\
(\mathrm{HFD}+\mathrm{RSV} \\
\text { versus } \mathrm{HFD}) \\
\end{array}$ & $\begin{array}{c}\text { Up/down } \\
(\mathrm{HFD}+\mathrm{RSV} \text { versus } \\
\text { HFD })\end{array}$ \\
\hline NONMMUT147944.1 & 7.361654305 & $3.08 E-05$ & $\mathrm{Up}$ & -7.65705 & $2.59 E-05$ & Down \\
\hline NONMMUT018494.2 & 6.726119826 & $2.72 E-30$ & $\mathrm{Up}$ & -6.63171 & $1.25 E-30$ & Down \\
\hline NONMMUT056862.2 & 6.372532912 & 0.000101 & $\mathrm{Up}$ & -6.47057 & 0.000104 & Down \\
\hline NONMMUT001029.2 & 5.951073546 & $1.73 E-06$ & $\mathrm{Up}$ & -6.20954 & $1.09 E-06$ & Down \\
\hline NONMMUT034722.2 & 5.925450571 & $5.74 E-05$ & $\mathrm{Up}$ & -5.99116 & $5.98 E-05$ & Down \\
\hline NONMMUT042491.2 & 5.439454581 & 0.000152 & $\mathrm{Up}$ & -6.0331 & $6.81 E-05$ & Down \\
\hline NONMMUT011659.2 & 5.319133003 & 0.000118 & Up & -5.39538 & 0.000122 & Down \\
\hline NONMMUT028972.2 & 5.109498067 & $9.40 E-05$ & $\mathrm{Up}$ & -5.33988 & $7.69 E-05$ & Down \\
\hline NONMMUT056994.2 & 4.330643092 & $1.11 E-07$ & $\mathrm{Up}$ & -3.97586 & $2.22 E-06$ & Down \\
\hline NONMMUT044528.2 & 4.3149951 & $2.45 E-05$ & $\mathrm{Up}$ & -4.67614 & $1.12 E-05$ & Down \\
\hline NONMMUT139818.1 & 4.27510251 & $5.42 E-10$ & $\mathrm{Up}$ & -2.93598 & $3.09 E-07$ & Down \\
\hline NONMMUT006490.2 & 4.040499067 & $4.37 E-05$ & $\mathrm{Up}$ & -4.25073 & $3.25 E-05$ & Down \\
\hline NONMMUT153460.1 & 3.987910041 & $1.14 E-10$ & $\mathrm{Up}$ & -4.43375 & $1.79 E-11$ & Down \\
\hline NONMMUT047957.2 & 3.622830463 & $5.31 E-12$ & $\mathrm{Up}$ & -2.17202 & $1.80 E-06$ & Down \\
\hline NONMMUT061044.2 & 3.580172631 & 0.000913 & $\mathrm{Up}$ & -5.02265 & 0.000116 & Down \\
\hline NONMMUT041793.2 & 3.348027654 & 0.000102 & $\mathrm{Up}$ & -4.15666 & $2.47 E-06$ & Down \\
\hline MSTRG.26789.5 & 3.346146359 & $4.90 E-17$ & $\mathrm{Up}$ & -2.35799 & $1.25 E-05$ & Down \\
\hline NONMMUT141647.1 & 3.045932847 & $2.40 E-05$ & $\mathrm{Up}$ & -3.7519 & $3.30 E-05$ & Down \\
\hline NONMMUT019242.2 & 2.994018555 & $1.34 E-16$ & $\mathrm{Up}$ & -1.86027 & $2.37 E-07$ & Down \\
\hline NONMMUT054892.2 & 2.304363454 & $8.76 E-08$ & $\mathrm{Up}$ & -1.99848 & $3.44 E-05$ & Down \\
\hline NONMMUT051479.2 & 2.213054794 & 0.00098 & Up & -3.05151 & $3.84 E-05$ & Down \\
\hline
\end{tabular}


TABle 3: Continued.

\begin{tabular}{|c|c|c|c|c|c|c|}
\hline LncRNA_id & $\begin{array}{c}\log 2 \mathrm{FC}(\mathrm{HFD} \\
\text { versus } \mathrm{CON})\end{array}$ & $\begin{array}{l}P \text { value }(\mathrm{HFD} \\
\text { versus } \mathrm{CON})\end{array}$ & $\begin{array}{c}\text { Up/down } \\
\text { (HFD versus } \\
\text { CON) }\end{array}$ & $\begin{array}{c}\log 2 \mathrm{FC} \\
(\mathrm{HFD}+\mathrm{RSV} \\
\text { versus HFD) } \\
\end{array}$ & $\begin{array}{c}P \text { value } \\
(\mathrm{HFD}+\mathrm{RSV} \\
\text { versus HFD) }\end{array}$ & $\begin{array}{c}\text { Up/down } \\
(\mathrm{HFD}+\mathrm{RSV} \text { versus } \\
\text { HFD })\end{array}$ \\
\hline NONMMUT003238.2 & 2.187198497 & 0.045286 & $\mathrm{Up}$ & -5.56027 & $8.43 E-05$ & Down \\
\hline NONMMUT004274.2 & 2.077962645 & $1.51 E-05$ & Up & -1.86821 & 0.000128 & Down \\
\hline NONMMUT006717.2 & 2.041245592 & $1.41 E-06$ & Up & -2.02194 & $8.66 E-06$ & Down \\
\hline ENSMUST00000145549 & 1.981088074 & $3.80 E-07$ & Up & -1.97828 & $7.99 E-07$ & Down \\
\hline NONMMUT071342.2 & 1.630473739 & 0.012079 & $\mathrm{Up}$ & -3.80688 & $1.60 E-13$ & Down \\
\hline NONMMUT005295.2 & 1.449638857 & $1.11 E-06$ & Up & -1.41758 & $2.03 E-05$ & Down \\
\hline NONMMUT070926.2 & 1.34488241 & 0.026106 & $\mathrm{Up}$ & -2.66561 & $8.38 E-08$ & Down \\
\hline NONMMUT006953.2 & 1.275954985 & 0.000623 & Up & -2.36716 & $2.40 E-09$ & Down \\
\hline NONMMUT044897.2 & 1.249501171 & $2.60 E-05$ & $\mathrm{Up}$ & -1.43116 & $2.19 E-05$ & Down \\
\hline NONMMUT048831.2 & -1.272978476 & 0.046708 & Down & 1.547263 & 0.000408 & Up \\
\hline NONMMUT018620.2 & -1.425732816 & 0.032203 & Down & 1.762165 & 0.000445 & Up \\
\hline NONMMUT051818.2 & -1.49981749 & 0.00019 & Down & 1.757541 & 0.000229 & Up \\
\hline NONMMUT148959.1 & -1.821849383 & 0.022286 & Down & 2.39635 & 0.000835 & $\mathrm{Up}$ \\
\hline NONMMUT025210.2 & -1.865766716 & 0.032229 & Down & 3.501751 & $1.41 E-09$ & Up \\
\hline NONMMUT024340.2 & -1.99805347 & $6.44 E-07$ & Down & 1.727535 & 0.000114 & Up \\
\hline NONMMUT030788.2 & -2.072643561 & 0.008364 & Down & 3.386966 & $6.32 E-08$ & Up \\
\hline NONMMUT083064.1 & -2.184391746 & 0.02567 & Down & 3.578567 & $9.44 E-07$ & $\mathrm{Up}$ \\
\hline NONMMUT117757.1 & -2.220356685 & 0.000126 & Down & 1.946318 & 0.000268 & Up \\
\hline NONMMUT145026.1 & -2.225432629 & 0.005502 & Down & 2.733938 & 0.00075 & $\mathrm{Up}$ \\
\hline NONMMUT143802.1 & -2.239123899 & 0.000257 & Down & 2.484837 & 0.000105 & Up \\
\hline NONMMUT081465.1 & -2.275670974 & 0.000759 & Down & 2.214893 & 0.000569 & Up \\
\hline NONMMUT071570.2 & -2.342779878 & $1.52 E-05$ & Down & 2.040383 & $7.76 E-05$ & $\mathrm{Up}$ \\
\hline NONMMUT145721.1 & -2.395813608 & 0.00904 & Down & 3.144452 & 0.000475 & Up \\
\hline NONMMUT082610.1 & -2.721997741 & 0.004713 & Down & 3.500026 & $3.04 E-06$ & $\mathrm{Up}$ \\
\hline NONMMUT032162.2 & -2.756781381 & 0.000975 & Down & 3.252807 & $1.74 E-06$ & Up \\
\hline NONMMUT098269.1 & -2.860045031 & $2.20 E-09$ & Down & 2.306565 & 0.000783 & $\mathrm{Up}$ \\
\hline NONMMUT119847.1 & -2.871001568 & $6.07 E-06$ & Down & 2.197726 & 0.000589 & Up \\
\hline NONMMUT004497.2 & -3.006495667 & $6.12 E-06$ & Down & 3.18202 & $2.60 E-06$ & $\mathrm{Up}$ \\
\hline NONMMUT144862.1 & -3.021747289 & 0.000339 & Down & 2.825872 & 0.000763 & Up \\
\hline NONMMUT152140.1 & -3.390813528 & 0.005418 & Down & 3.671173 & 0.000159 & $\mathrm{Up}$ \\
\hline NONMMUT145717.1 & -3.429960838 & 0.003878 & Down & 4.82329 & $1.67 E-05$ & Up \\
\hline ENSMUST00000181045 & -3.5774189 & $2.18 E-05$ & Down & 4.111429 & $1.20 E-07$ & $\mathrm{Up}$ \\
\hline MSTRG.8668.1 & -3.650059914 & $8.30 E-13$ & Down & 2.418835 & 0.0002 & Up \\
\hline NONMMUT145909.1 & -4.44549442 & $6.50 E-05$ & Down & 3.575655 & $3.04 E-05$ & Up \\
\hline NONMMUT008421.2 & -4.495334741 & 0.000753 & Down & 5.182219 & $4.44 E-06$ & $\mathrm{Up}$ \\
\hline ENSMUST00000161890 & -5.096061065 & 0.013794 & Down & 6.206952 & $7.53 E-05$ & $\mathrm{Up}$ \\
\hline NONMMUT128951.1 & -5.845021433 & $2.88 E-07$ & Down & 4.876355 & $2.89 E-05$ & $\mathrm{Up}$ \\
\hline NONMMUT065156.2 & -7.177240343 & $5.91 E-41$ & Down & 6.653339 & $2.22 E-10$ & Up \\
\hline NONMMUT026869.2 & -8.335434963 & $1.59 E-11$ & Down & 7.485915 & $1.33 E-06$ & Up \\
\hline
\end{tabular}

Top 30 upregulated or downregulated lncRNAs in three groups. HFD: high-fat diet; CON: control; RSV: resveratrol; lncRNA: long noncoding RNA.

TABle 4: Top 30 significantly differential expression mRNAs in mice.

\begin{tabular}{lcccccc}
\hline Gene name & $\begin{array}{c}\text { log2FC (HFD } \\
\text { versus CON) }\end{array}$ & $\begin{array}{c}P \text { value (HFD } \\
\text { versus CON) }\end{array}$ & $\begin{array}{c}\text { Up/down } \\
\text { (HFD versus } \\
\text { CON) }\end{array}$ & $\begin{array}{c}\text { log2FC (HFD + RSV } \\
\text { versus HFD) }\end{array}$ & $\begin{array}{c}P \text { value (HFD + RSV } \\
\text { versus HFD) }\end{array}$ & $\begin{array}{c}\text { Up/down } \\
\text { (HFD + RSV versus } \\
\text { HFD) }\end{array}$ \\
\hline Gm49388 & 6.06138 & $1.92 E-10$ & $\mathrm{Up}$ & -2.53021 & 0.044372 & Down \\
Gm26876 & 3.811306 & $7.03 E-09$ & $\mathrm{Up}$ & -3.11381 & $5.15 E-07$ & Down \\
4930512H18Rik & 3.787461 & $3.47 E-18$ & $\mathrm{Up}$ & -1.20868 & 0.003987 & Down \\
Gm29676 & 3.283294 & 0.00530139 & $\mathrm{Up}$ & -3.86133 & 0.002862 & Down \\
Map6d1 & 3.074208 & 0.007729399 & $\mathrm{Up}$ & -1.72651 & 0.047048 & Down \\
Ppm1n & 3.000682 & $2.93 E-08$ & $\mathrm{Up}$ & -1.05825 & 0.012598 & Down \\
Sh3gl2 & 2.803522 & $1.83 E-09$ & $\mathrm{Up}$ & -2.08107 & $5.64 E-06$ & Down \\
Clca4a & 2.729295 & 0.000758257 & $\mathrm{Up}$ & -2.14669 & 0.002907 & Down \\
Gm49347 & 2.69398 & 0.016676861 & $\mathrm{Up}$ & -2.79388 & 0.016914 & Down \\
Gm33543 & 2.661545 & $2.23 E-13$ & $\mathrm{Up}$ & -1.30568 & 0.029604 & Down \\
Ccl12 & 2.641596 & 0.005800303 & $\mathrm{Up}$ & -3.96508 & 0.00141 & Down \\
\hline
\end{tabular}


TABLE 4: Continued.

\begin{tabular}{|c|c|c|c|c|c|c|}
\hline Gene name & $\begin{array}{l}\log 2 \mathrm{FC}(\mathrm{HFD} \\
\text { versus } \mathrm{CON})\end{array}$ & $\begin{array}{l}P \text { value }(\mathrm{HFD} \\
\text { versus } \mathrm{CON})\end{array}$ & $\begin{array}{c}\text { Up/down } \\
\text { (HFD versus } \\
\text { CON) } \\
\end{array}$ & $\begin{array}{c}\log 2 \mathrm{FC}(\mathrm{HFD}+\mathrm{RSV} \\
\text { versus HFD })\end{array}$ & $\begin{array}{c}P \text { value }(H F D+R S V \\
\text { versus } H F D)\end{array}$ & $\begin{array}{c}\text { Up/down } \\
(\mathrm{HFD}+\mathrm{RSV} \text { versus } \\
\text { HFD }) \\
\end{array}$ \\
\hline Gm9402 & 2.633552 & 0.044305865 & $\mathrm{Up}$ & -2.95627 & 0.046539 & Down \\
\hline Gm48719 & 2.618363 & $1.87 E-05$ & Up & -1.43542 & 0.008835 & Down \\
\hline Rpl21-ps12 & 2.537707 & 0.000902295 & Up & -1.83961 & 0.012602 & Down \\
\hline Gm15478 & 2.466703 & $1.04 E-05$ & Up & -1.16718 & 0.029683 & Down \\
\hline Kcnh7 & 2.453351 & $3.86 E-11$ & Up & -1.11192 & 0.016375 & Down \\
\hline Socs1 & 2.44948 & $4.05 E-07$ & Up & -2.36143 & $1.61 E-06$ & Down \\
\hline Gm47603 & 2.39615 & $2.51 E-05$ & Up & -1.36179 & 0.007302 & Down \\
\hline C130026L21Rik & 2.31491 & 0.023709939 & Up & -2.52323 & 0.024031 & Down \\
\hline AY036118 & 2.26368 & 0.014427254 & Up & -2.08364 & 0.022577 & Down \\
\hline Cish & 2.1587 & $1.43 E-14$ & $\mathrm{Up}$ & -2.48736 & $1.64 E-20$ & Down \\
\hline $\mathrm{Cd} 209 \mathrm{e}$ & 2.146486 & 0.001095503 & Up & -1.52077 & 0.033734 & Down \\
\hline Dkk3 & 2.060882 & $1.16 E-07$ & $\mathrm{Up}$ & -1.51887 & 0.000588 & Down \\
\hline Pou2f3 & 2.046599 & 0.032113693 & Up & -2.67227 & 0.010465 & Down \\
\hline Pcsk1 & 1.960889 & $6.87 E-07$ & Up & -1.14143 & 0.001394 & Down \\
\hline Gm26635 & 1.857674 & 0.020348988 & Up & -1.53523 & 0.040218 & Down \\
\hline B230312C02Rik & 1.846494 & $1.97 E-10$ & Up & -1.85503 & $2.38 E-10$ & Down \\
\hline 4932438H23Rik & 1.740581 & 0.019356615 & Up & -1.8725 & 0.019401 & Down \\
\hline $\mathrm{Ccl} 7$ & 1.669165 & 0.006830603 & Up & -1.92417 & 0.005174 & Down \\
\hline Megf11 & 1.597213 & 0.002069216 & $\mathrm{Up}$ & -1.26422 & 0.028085 & Down \\
\hline Sox10 & -1.00199 & 0.00499603 & Down & 1.277467 & 0.008898 & $\mathrm{Up}$ \\
\hline Gm17971 & -1.01911 & 0.001132241 & Down & 1.082473 & 0.012236 & Up \\
\hline Mgat3 & -1.02613 & 0.002140658 & Down & 1.038285 & 0.007315 & Up \\
\hline Gas213 & -1.02807 & 0.020453198 & Down & 1.139046 & 0.006303 & Up \\
\hline Bcas1 & -1.04182 & 0.015404068 & Down & 1.7179 & 0.002295 & Up \\
\hline Asphd2 & -1.04528 & 0.020154215 & Down & 1.010603 & 0.025998 & Up \\
\hline Gm37537 & -1.05529 & 0.004647624 & Down & 1.013631 & 0.014094 & Up \\
\hline Fos & -1.07258 & 0.000205959 & Down & 1.126982 & 0.002405 & $\mathrm{Up}$ \\
\hline Gldn & -1.07534 & 0.029747688 & Down & 1.119705 & 0.011748 & Up \\
\hline Pmp22 & -1.10369 & 0.0243704 & Down & 1.272211 & 0.007779 & Up \\
\hline Sptbn5 & -1.10708 & 0.002406818 & Down & 1.274 & 0.003898 & Up \\
\hline Plekha4 & -1.11677 & 0.003070017 & Down & 1.306669 & 0.002928 & $\mathrm{Up}$ \\
\hline Kcna6 & -1.11915 & 0.010919737 & Down & 1.15063 & 0.011805 & Up \\
\hline Gabrr1 & -1.12133 & 0.007296347 & Down & 1.004214 & 0.027277 & $\mathrm{Up}$ \\
\hline Mir1904 & -1.12175 & 0.004769582 & Down & 1.111331 & 0.011967 & Up \\
\hline Kif19a & -1.14672 & 0.006248502 & Down & 1.502258 & 0.001265 & Up \\
\hline Fosl2 & -1.16247 & $1.85 E-08$ & Down & 1.02334 & $4.21 E-05$ & Up \\
\hline Lrrc71 & -1.16508 & 0.023094219 & Down & 1.001178 & 0.048202 & Up \\
\hline Fhl4 & -1.1723 & 0.034470511 & Down & 1.121767 & 0.045133 & Up \\
\hline $\mathrm{Fa} 2 \mathrm{~h}$ & -1.18303 & 0.034450796 & Down & 1.299243 & 0.029667 & Up \\
\hline Gm37510 & -1.19184 & 0.004453269 & Down & 1.03012 & 0.008302 & Up \\
\hline Ppia & -1.19672 & 0.001831193 & Down & 1.119291 & $2.62 E-05$ & Up \\
\hline Tnfsf $13 b$ & -1.20579 & 0.016517031 & Down & 1.142204 & 0.010129 & Up \\
\hline Tox & -1.21833 & 0.015176461 & Down & 1.414292 & 0.001254 & Up \\
\hline Rasgef1c & -1.2224 & 0.036363327 & Down & 1.405989 & 0.013403 & Up \\
\hline $\mathrm{Mt} 3$ & -1.22738 & 0.007211092 & Down & 1.291177 & 0.005362 & Up \\
\hline Elovl7 & -1.24473 & 0.016851444 & Down & 1.412536 & 0.009919 & Up \\
\hline Wnt2b & -1.26686 & 0.007582771 & Down & 1.527884 & 0.008061 & Up \\
\hline Tenm2 & -1.27034 & 0.005050296 & Down & 1.05799 & 0.010664 & Up \\
\hline Mmp27 & -1.27782 & 0.027735632 & Down & 1.191382 & 0.04508 & Up \\
\hline
\end{tabular}

The table lists the top 30 of the results for mRNA with upregulation or downregulation in expression in three groups. HFD: high-fat diet; CON: control; RSV: resveratrol; lncRNA: long noncoding RNA.

and without $0.25 \mathrm{mM}$ PA. Glucose concentrations were determined at $0,8,16$, and $24 \mathrm{~h}$. There was no significant difference in control and PA groups at 0,8 , and $16 \mathrm{~h}$; however, the glucose concentration of the PA group was distinctly elevated at $24 \mathrm{~h}$ compared with that of the control group, thereby indicating that the IR model was successfully established (Figure 5(a)). Besides, the glucose concentration was obviously decreased at $24 \mathrm{~h}$ in the RSV group (Figure 5(b)).

The cell survival rate of $\mathrm{C} 2 \mathrm{C} 12$ cells $24 \mathrm{~h}$ after RSV administration was approximately $30-100 \mu \mathrm{m}$. The results showed that $30 \mu \mathrm{m}$ of RSV had no significant influence on 


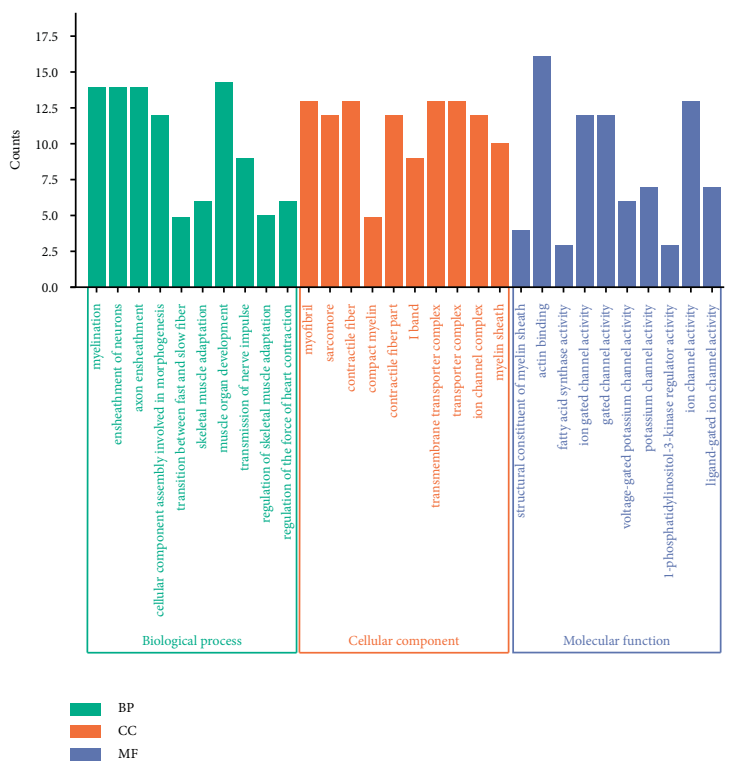

(a)

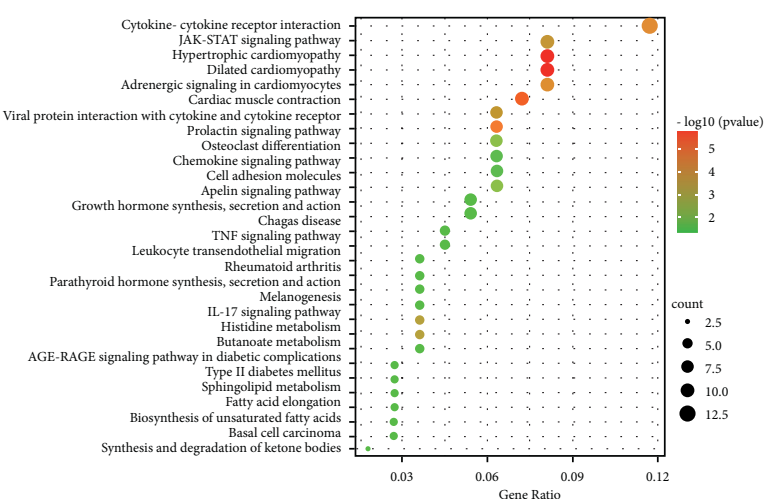

(b)

Figure 3: Gene Ontology (GO) and Kyoto Encyclopedia of Genes and Genomes (KEGG) pathway analysis of differentially expressed genes in the three groups. (a) Top 30 GO terms related to differentially expressed mRNAs. (b) Top 30 KEGG pathways related to differentially expressed mRNAs.

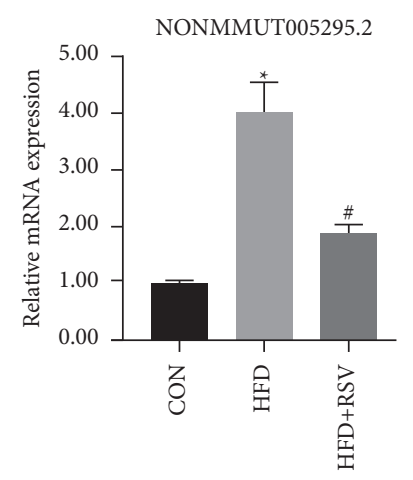

(a)

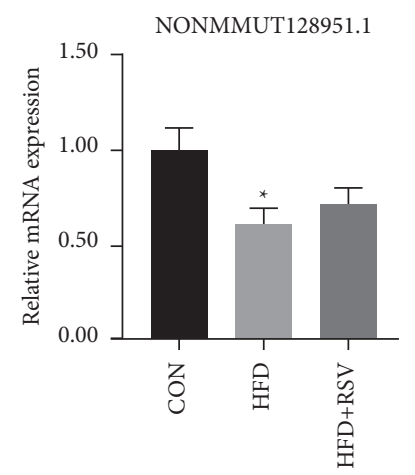

(c)

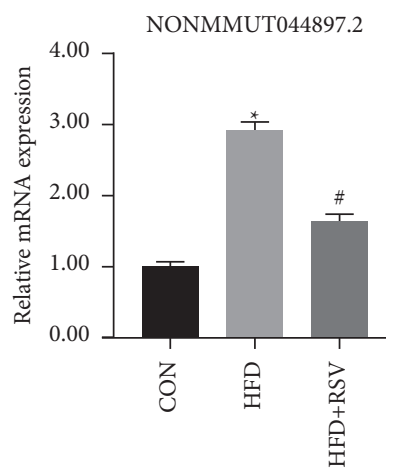

(b)

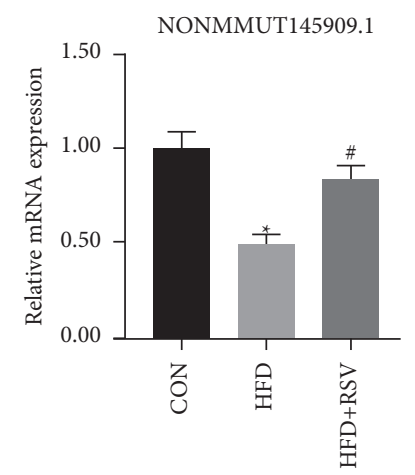

(d)

FIgUre 4: Continued. 


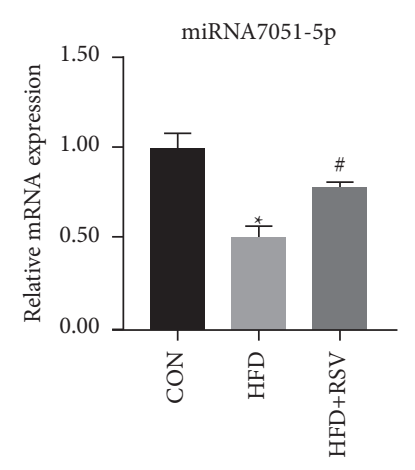

(e)

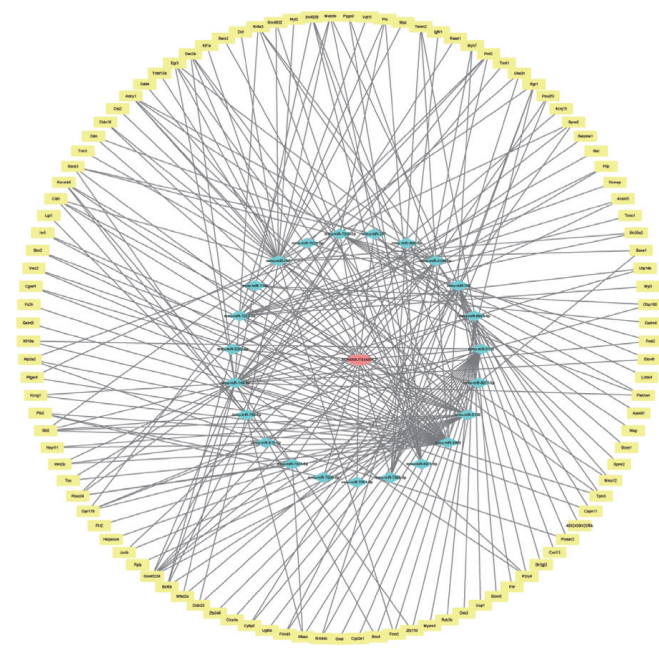

(g)

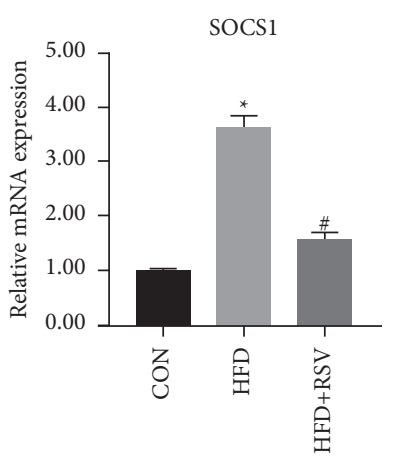

(f)

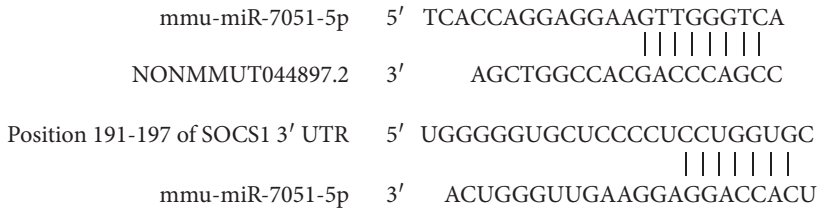

(h)

Figure 4: Validation of lncRNAs by RT-qPCR in vivo. Expression of NONMMUT005295.2 (a), NONMMUT044897.2 (b), NONMMUT128951.1 (c), NONMMUT145909.1 (d), miRNA-7051-5p mRNA (e), and SOCS1 mRNA (f) in different groups. (g) The NONMMUT044897.2 lncRNA-miRNA-mRNA network. (h) The positions of miR-7051-5p binding sites on NONMMUT044897.2 and the positions of miR-7051-5p binding sites on SOCS1. Data are expressed as the mean $\pm \mathrm{SD}(n=6) .{ }^{*} P<0.05$ versus CON; ${ }^{\#} P<0.05$ versus HFD.

the survival rate of $\mathrm{C} 2 \mathrm{C} 12$ cells (Figure 5(c)). The cell survival rate of the PA group (84\%) was lower than that of the control (89.6\%), while that of the $30 \mu \mathrm{M} \mathrm{PA}+\mathrm{RSV}$ group (85\%) was higher than that of the PA group. However, there were no statistical differences between these three groups (Figure 5(d)).

4.7. RT-qPCR Validation In Vitro. Two lncRNAs were upregulated in PA in which downregulation of PA + RSV (NONMMUT044897.2; NONMMUT139818.1) and upregulation of three lncRNAs (NONMMUT071570.2; NONMMUT065156.2; NONMMUT00000181045) were observed. The expression levels of the selected lncRNAs were consistent with those of the sequencing results (Figure 6(a)6(e)), but there was no statistical difference in the increase of NONMMUT00000181045 in the PA + RSV group. To verify the relationship between NONMMUT044897.2 and RSV in vitro, $\mathrm{C} 2 \mathrm{C} 12$ cells were transfected with lentivirus. The results of this transfection showed that, compared with the control group, NONMMUT044897.2 expression was robustly increased in the PA and PA + shRNA-NC groups. The
PA + shRNA-NONMMUT044897.2 group had a decreased expression of NONMMUT044897.2 compared with that of the PA group. RSV administration also resulted in a decrease in the expression of NONMMUT044897.2 compared with that of the PA group (Figure 6(g)).

Relative to the control group, miR-7051-5p mRNA expression in the PA and PA + shRNA-NC groups was significantly reduced, while knockdown of NONMMUT044897.2 and RSV treatment markedly increased the miR-7051-5p mRNA expression level (Figure 6(f)). The concentration of glucose in the media of the PA and PA + shRNA-NC groups was substantially increased compared with that of the control group. NONMMUT044897.2 silencing and RSV treatment strikingly overturned the glucose concentrations in the medium (Figure 6(h)). Knockdown of NONMMUT044897.2 distinctively upregulated the p-AKT, p-GSK3 $\beta$, and GLUT4 protein levels and greatly reduced the SOCS1 protein level, compared with those of the PA group. RSV treatment had a similar effect in terms of NONMMUT044897.2 silencing on p-AKT, p-GSK3 $\beta$, GLUT4, and SOCS1 protein levels (Figures 6(i)-6(o)). 


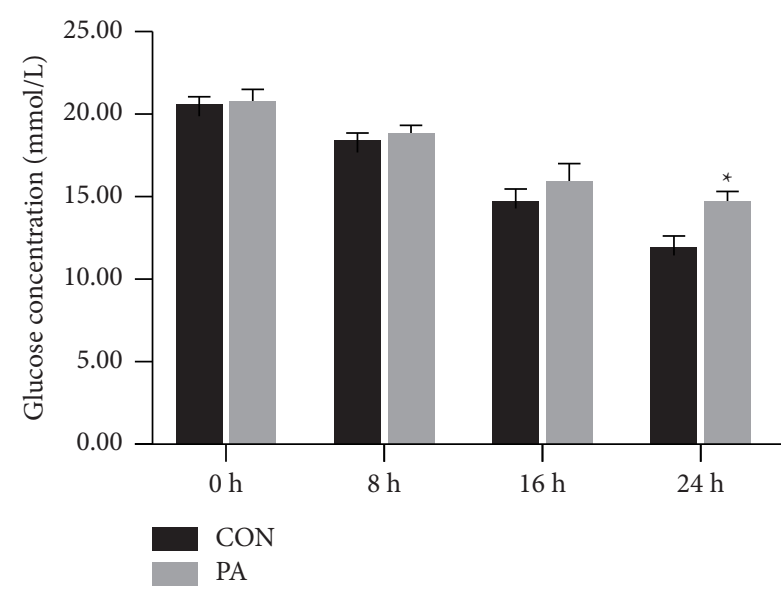

(a)

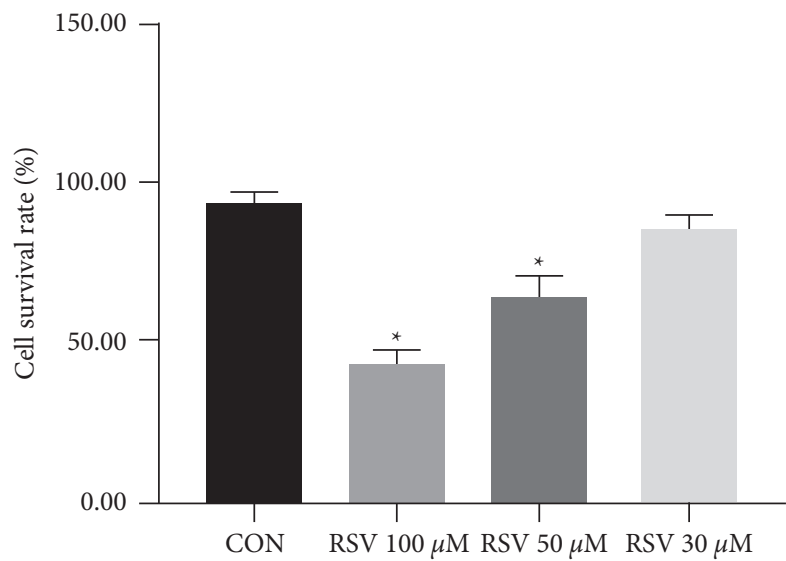

(c)

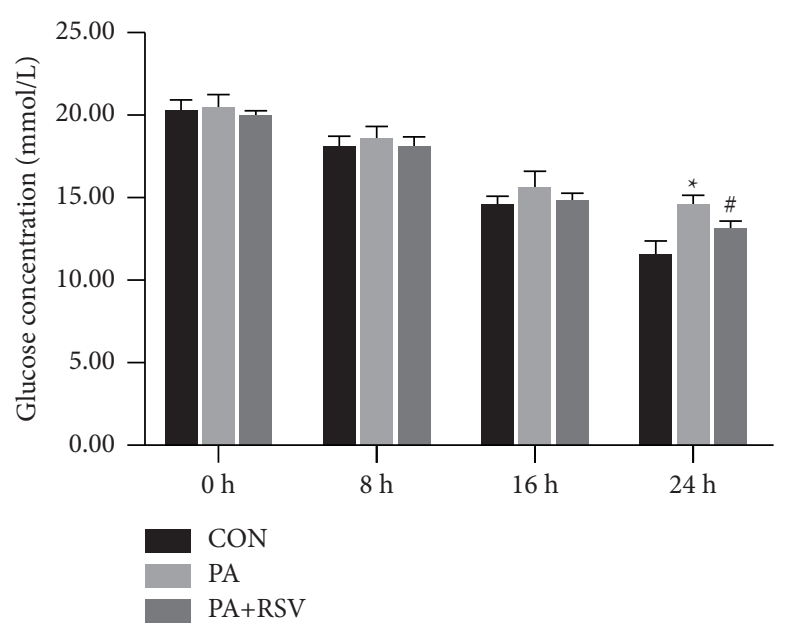

(b)

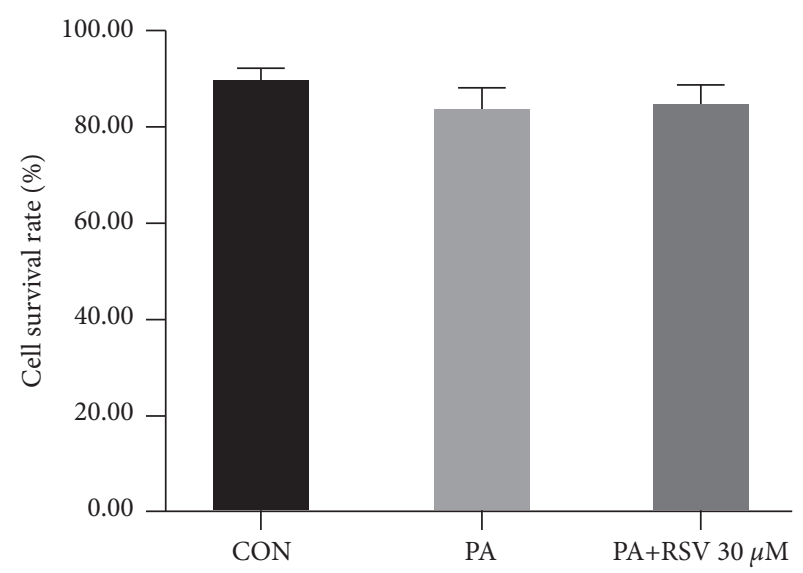

(d)

FigURE 5: Resveratrol reduced PA-induced glucose concentration in vitro. Glucose concentration in the medium after $0,8,16$, and $24 \mathrm{~h}$ treatment with PA (a) and treatment with PA and RSV (b). Cell survival rate after $24 \mathrm{~h}$ treatment with different concentrations of RSV (c) or PA and RSV treatments (d). Data are shown as the mean \pm SD $(n=6) .{ }^{*} P<0.05$ versus CON; ${ }^{\#} P<0.05$ versus PA.

\section{Discussion}

A large number of experiments have verified the therapeutic effect of RSV in IR [32, 33]. In this study, we demonstrated that RSV influenced the amelioration of IR in HFD-fed mice. These findings have also been reported by several studies $[11,12]$. In the HFD + RSV group, blood glucose, insulin index, blood lipids, and area under the curve were decreased. RSV treatment ameliorated HFD-induced IR in mice by restoring the insulin signaling pathway gene expression. After intervention with RSV, the protein expression levels of p-AKT, p-GSK3 $\beta$, and GLUT4 increased significantly.

High-throughput sequencing showed that there were 3,276 differentially expressed lncRNAs and 2,118 differentially expressed mRNAs in the HFD+RSV group, as compared with those in the control group, which yielded 1,640 differentially expressed lncRNAs and 604 differentially expressed mRNAs. We further found $338 \mathrm{mRNAs}$ and 629 lncRNAs whose expression was reversed in the HFD and the HFD + RSV groups, suggesting that RSV plays a significant role in the overall alteration of skeletal muscle gene expression. Moreover, RT-qPCR results revealed that RSV ameliorated IR by regulating the expression of lncRNAs in skeletal muscles. As mentioned above, the verified lncRNAs were consistent with those of the sequencing results. In addition, NONMMUT044897.2 was highly expressed. The KEGG analysis uncovered that the differential genes were part of T2DM. We found that NONMMUT044897.2 was associated with SOCS1, which was critically involved in T2DM. Overexpression of SOCS1 aggravated IR [5], which played an important role in T2DM. Hence, we selected this lncRNA for further study, which has not been reported before.

SOCS1 is a specific negative regulator that regulates the JAK/STAT pathway [34]. The expression of SOCS1 increases with IR and decreases with the phosphorylation of IRS-1. Overexpression of SOCS-1 could inhibit insulin-induced glycogen synthesis in L6 myotubes [6]. AKT has essential roles in many signaling pathways, such as cell survival and cell metabolism. AKT is the center of the insulin signaling pathway, which regulates glucose and lipid metabolism. Activated AKT can stimulate the translocation of insulinsensitive GLUT4 to the cell membrane through its 


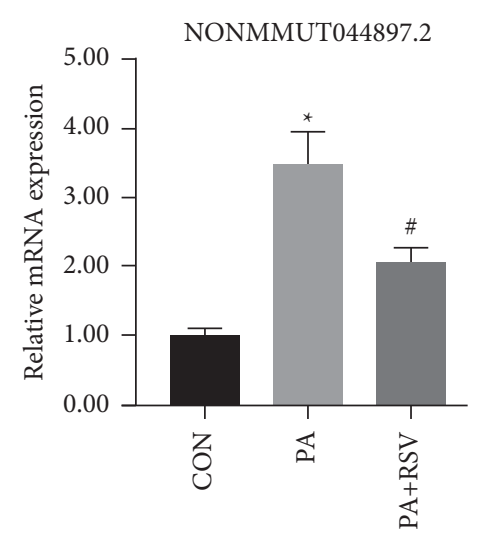

(a)

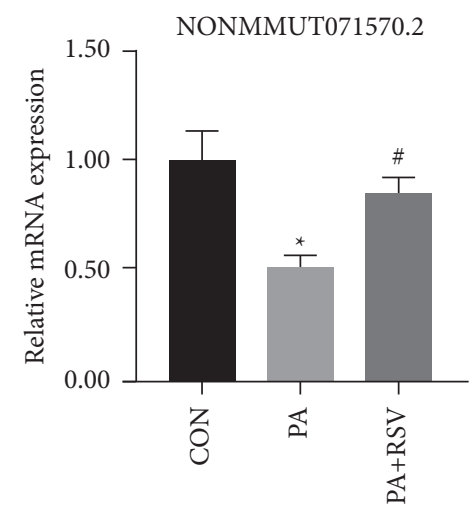

(d)

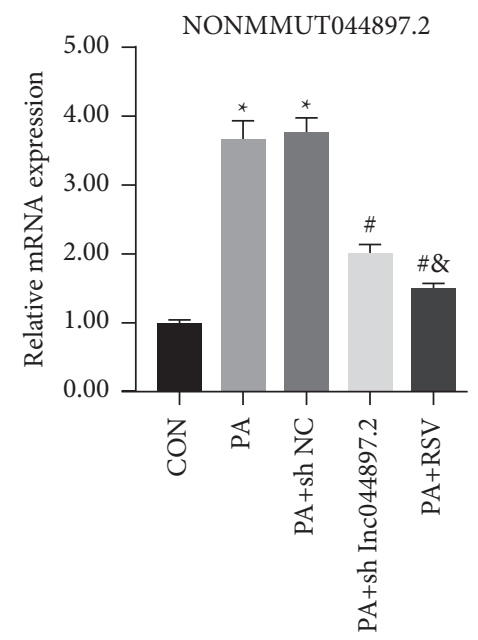

(g)

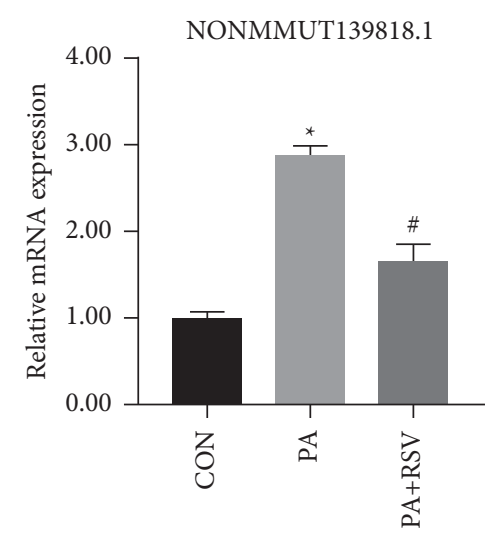

(b)

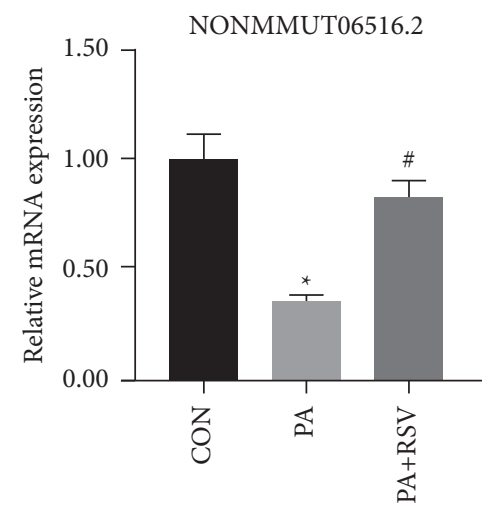

(e)

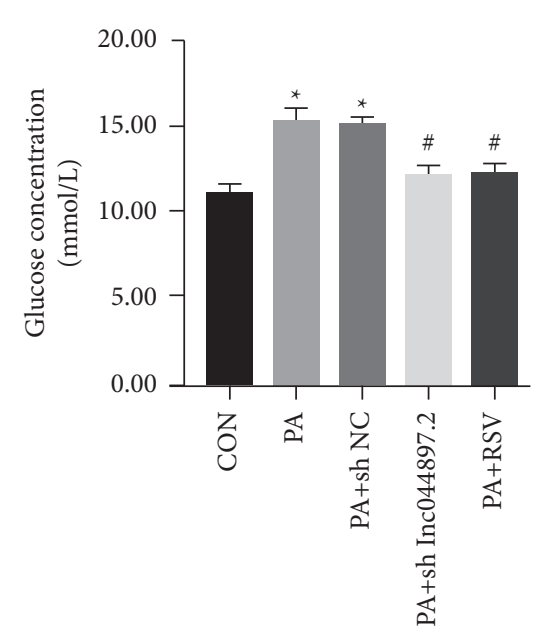

(h)

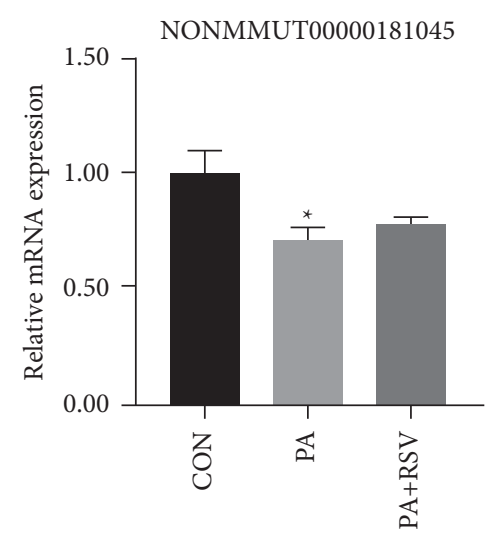

(c)

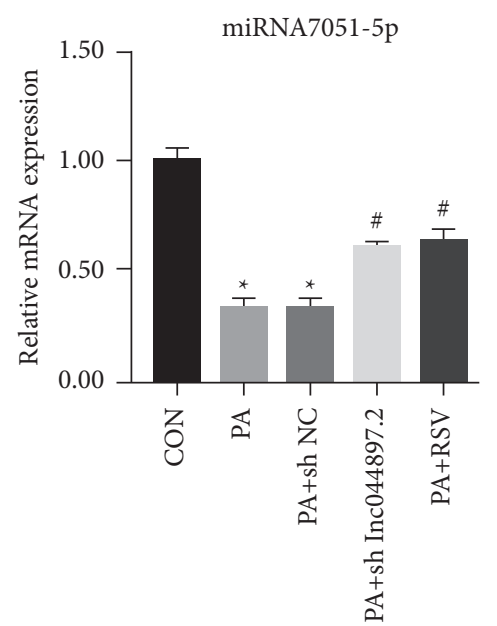

(f)

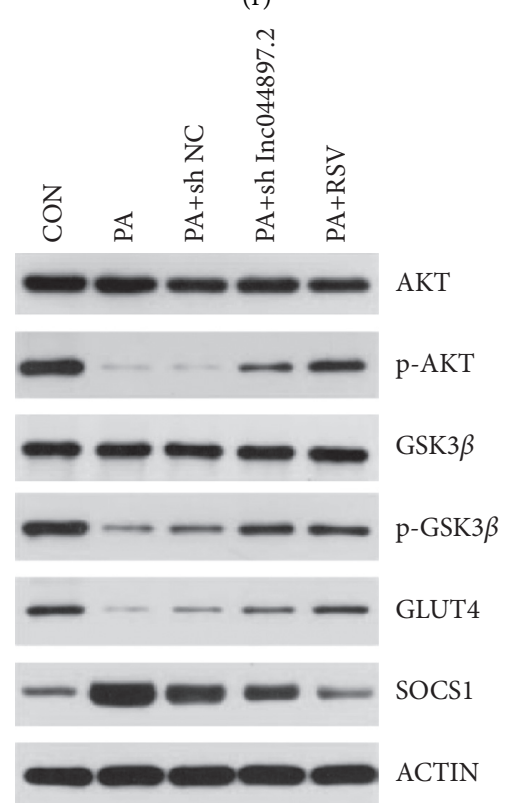

(i)

Figure 6: Continued. 


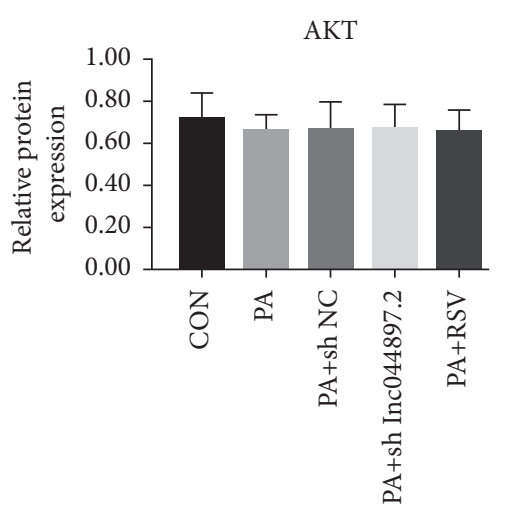

(j)

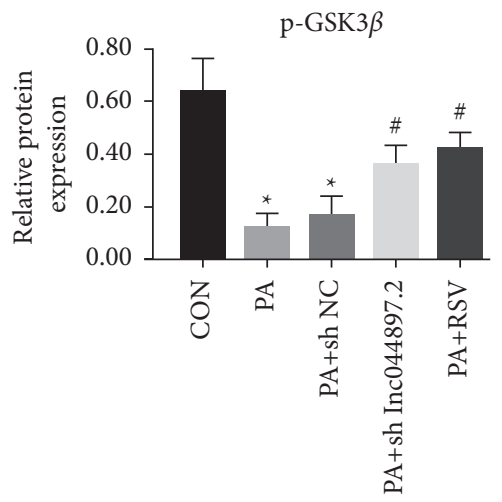

$(\mathrm{m})$

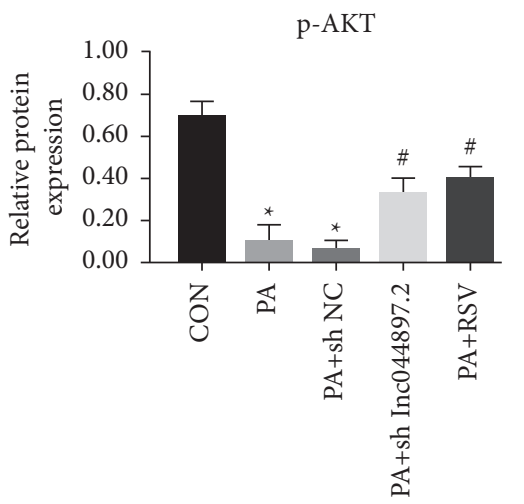

$(\mathrm{k})$

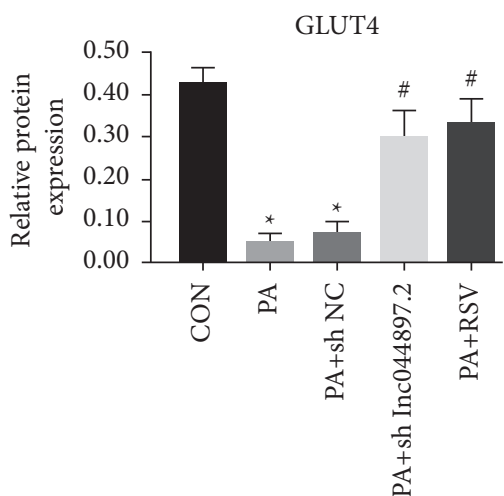

(n)

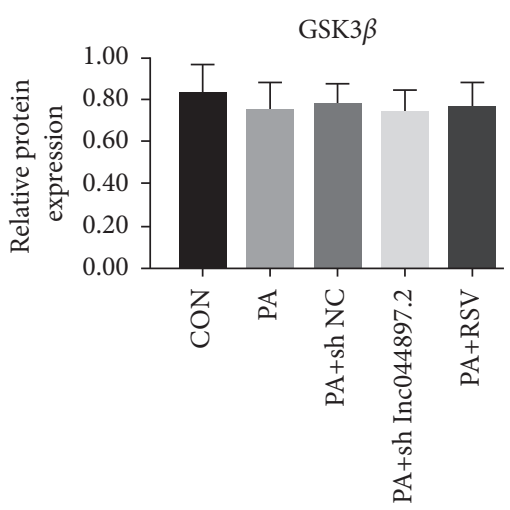

(1)

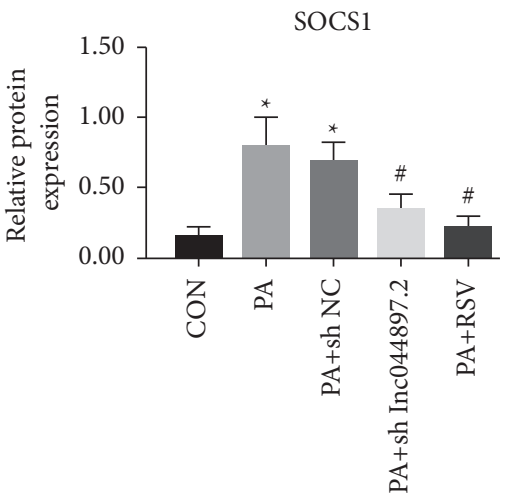

(o)

FIgURE 6: Resveratrol improved skeletal muscle insulin resistance by downregulating the lncRNA NONMUT044897.2 in vitro. Validation of lncRNAs NONMMUT044897.2 (a), NONMMUT139818.1 (b), NONMMUT00000181045(c), NONMMUT071570.2 (d), and NONMMUT06516.2 (e) by RT-qPCR. Expression of miR-7051-5p mRNA (f) and NONMMUT044897.2 mRNA (g) after shRNA transfection into $\mathrm{C} 2 \mathrm{C} 12$ cells in different groups. (h) Glucose concentrations in the culture medium after shRNA transfection into C2C12 cells in different groups. (i) Protein bands of insulin signaling pathway-related molecules. Densitometric analysis of (j) AKT, (k) p-AKT, (l) GSK3 $\beta$, (m) p-GSK3 $\beta$, (n) GLUT4, and (o) SOCS1. Data are presented as the mean \pm SD $(n=6) .{ }^{*} P<0.05$ versus CON, ${ }^{\#} P<0.05$ versus $\mathrm{PA}$, and ${ }^{\delta} \mathrm{P}<0.05$ versus $\mathrm{PA}+$ shRNA-NONMMUT044897.2.

downstream substrate AS160 to increase glucose uptake. It can also phosphorylate GSK3 $\beta$ to inhibit its activity, promote glycogen synthesis, lower blood sugar, and improve IR [35]. Furthermore, an HFD could result in the decrease of skeletal muscle IRS-1, P13K, AKT, and GLUT4 gene expression levels and reduce $\mathrm{p}-\mathrm{AKT}$ (ser473) and $\mathrm{p}-\mathrm{GSK} 3 \beta$ protein expression levels. Studies have reported that overexpression of SOCS1 may inhibit the phosphorylation and activation of IRS-1 $[6,34]$, which in turn inhibits the activation of AKT, thereby indicating that there is an important link between AKT and SOCS1. We found that, in the IR model mice, mRNA and protein expression levels of SOCS1 were significantly increased, while RSV treatment exhibited the reverse trend, thereby improving IR and decreasing blood glucose levels.

Numerous studies have demonstrated that lncRNAs may be involved in human diseases by regulating the expression of miRNAs $[36,37]$. The ceRNA-network diagram uncovered the possible regulatory roles of candidate lncRNAs. NONMMUT044897.2 could regulate SOCS1 through two different miRNAs. To further clarify the relationship between NONMMUT044897.2 and SOCS1, we constructed NONMMUT044897.2 and miR-7051-5p, miR7051-5p, and SOCS1 base-pairing maps based on
NonCode, miRBase, and TargetScan databases. These results indicate that NONMMUT044897.2 might have regulated the expression of SOCS1 through miR-7051-5p. Future studies should perform luciferase assays to verify the interactions between NONMMUT044897.2, miR-7051$5 \mathrm{p}$, and SOCS1.

We confirmed that knockdown of NONMMUT044897.2 increased miR-7051-5p levels and promoted the expression of genes that are involved in the insulin signaling pathways (p-AKT, p-GSK3 $\beta$, and GLUT4). Meanwhile, the expression of SOCS1 was suppressed by silencing NONMMUT044897.2. Moreover, knockdown of NONMMUT044897.2 has led to reduced glucose concentration, which is similar to the phenotypes induced with RSV treatment. This indicates that RSV improves skeletal muscle IR through downregulating the lncRNA NONMM UT044897.2.

\section{Conclusions}

This research profiled the differential expression of lncRNAs between the IR model mice and those treated with RSV. We further revealed the potentially regulated lncRNA 
NONMMUT044897.2. More work remains to be done to prove the relationship between the NONMMUT044897.2/ miR-7051-5p/SOCS1 and RSV in the IR model. The study also has several limitations. First, in vivo animal models are needed to further silence NONMMUT044897.2 to verify the influence on IR. Second, the overexpression of NONMMUT044897.2 should be performed in vitro. Third, knockdown of the NONMMUT044897.2 in the RSV group should be done to observe its influence on the IR model. Overall, our data indicated that RSV could promote skeletal muscle IR, at least partially, via a lncRNA NONMMUT044897.2/miR-7051-5p/SOCS1 pathway. This provides a new perspective for the RSV treatment of IR in skeletal muscles.

\section{Data Availability}

The datasets used and/or analyzed during the current study are available from the corresponding author on reasonable request.

\section{Disclosure}

This article has been preprinted at https://www. researchsquare.com/article/rs-315719/v1.

\section{Conflicts of Interest}

The authors declare that there are no conflicts of interest.

\section{Acknowledgments}

The authors sincerely thank the teachers at the Clinical Medical Research Centre of Hebei General Hospital who helped them during the experiment. This study was supported by a grant from the Natural Science Foundation of Hebei Province (no. H201830-7071).

\section{Supplementary Materials}

All differentially expressed lncRNAs and mRNAs in the control, HFD, and HFD + RSV groups are listed in Supplementary Tables S1 and S2. (Supplementary Materials)

\section{References}

[1] M. P. Czech, "Insulin action and resistance in obesity and type 2 diabetes," Nature Medicine, vol. 23, no. 7, pp. 804-814, 2017.

[2] K. E. Merz and D. C. Thurmond, "Role of skeletal muscle in insulin resistance and glucose uptake," Comprehensive Physiology, vol. 10, no. 3, pp. 785-809, 2011.

[3] B. R. Sharma, H. J. Kim, and D. Y. Rhyu, "Caulerpa lentillifera extract ameliorates insulin resistance and regulates glucose metabolism in C57BL/KsJ-db/db mice via PI3K/AKT signaling pathway in myocytes," Journal of Translational Medicine, vol. 13, no. 1, p. 62, 2015.

[4] H. Xu, Y. Zhou, Y. Liu et al., "Metformin improves hepatic IRS2/PI3K/Akt signaling in insulin-resistant rats of NASH and cirrhosis," Journal of Endocrinology, vol. 229, no. 2, pp. 133-144, 2016.
[5] K. Ueki, T. Kadowaki, and C. Kahn, "Role of suppressors of cytokine signaling SOCS-1 and SOCS-3 in hepatic steatosis and the metabolic syndrome," Hepatology Research, vol. 33, no. 2, pp. 185-192, 2005.

[6] L. Rui, M. Yuan, D. Frantz, S. Shoelson, and M. F. White, "SOCS-1 and SOCS-3 block insulin signaling by ubiquitinmediated degradation of IRS1 and IRS2," Journal of Biological Chemistry, vol. 277, no. 44, pp. 42394-42398, 2002.

[7] P. Palsamy and S. Subramanian, "Resveratrol, a natural phytoalexin, normalizes hyperglycemia in streptozotocinnicotinamide induced experimental diabetic rats," Biomedicine \& Pharmacotherapy, vol. 62, no. 9, pp. 598-605, 2008.

[8] S. Gómez-Zorita, M. González-Arceo, J. Trepiana et al., "Comparative effects of pterostilbene and its parent compound resveratrol on oxidative stress and inflammation in steatohepatitis induced by high-fat high-fructose feeding," Antioxidants, vol. 9, no. 11, p. 1042, 2020.

[9] A. P. Singh, R. Singh, S. S. Verma et al., "Health benefits of resveratrol: evidence from clinical studies," Medicinal Research Reviews, vol. 39, no. 5, pp. 1851-1891, 2019.

[10] C. Carpéné, F. Les, G. Cásedas et al., "Resveratrol anti-obesity effects: rapid inhibition of adipocyte glucose utilization," Antioxidants, vol. 8, no. 3, p. 74, 2019.

[11] G. Luo, B. Huang, X. Qiu et al., "Resveratrol attenuates excessive ethanol exposure induced insulin resistance in rats via improving NAD+/NADH ratio," Molecular Nutrition \& Food Research, vol. 61, no. 11, Article ID 1700087, 2017.

[12] D. J. Den Hartogh, F. Vlavcheski, A. Giacca, and E. Tsiani, "Attenuation of free fatty acid (FFA)-induced skeletal muscle cell insulin resistance by resveratrol is linked to activation of AMPK and inhibition of mTOR and p70 S6K," International Journal of Molecular Sciences, vol. 21, no. 14, p. $4900,2020$.

[13] F. Vlavcheski, D. J. Den Hartogh, A. Giacca, and E. Tsiani, "Amelioration of high-insulin-induced skeletal muscle cell insulin resistance by resveratrol is linked to activation of AMPK and restoration of GLUT4 translocation," Nutrients, vol. 12, no. 4, p. 914, 2020.

[14] Y. J. Zhang, H. Zhao, L. Dong et al., "Resveratrol ameliorates high-fat diet-induced insulin resistance and fatty acid oxidation via ATM-AMPK axis in skeletal muscle," European Review for Medical and Pharmacological Sciences, vol. 23, pp. 9117-9125, 2019.

[15] Z. Tan, L.-J. Zhou, P.-W. Mu et al., "Caveolin-3 is involved in the protection of resveratrol against high-fat-diet-induced insulin resistance by promoting GLUT4 translocation to the plasma membrane in skeletal muscle of ovariectomized rats," The Journal of Nutritional Biochemistry, vol. 23, no. 12, pp. 1716-1724, 2012.

[16] Y. Y. Quan, S. Hua, W. Li, M. Zhan, Y. Li, and L. Lu, "Resveratrol bidirectionally regulates insulin effects in skeletal muscle through alternation of intracellular redox homeostasis," Life Sciences, vol. 242, Article ID 117188, 2020.

[17] J. J. Quinn and H. Y. Chang, "Unique features of long noncoding RNA biogenesis and function," Nature Reviews Genetics, vol. 17, no. 1, pp. 47-62, 2016.

[18] F. Constanty and A. Shkumatava, "lncRNAs in development and differentiation: from sequence motifs to functional characterization," Development, vol. 148, Article ID 182741, 2021.

[19] R. Thapar, J. L. Wang, M. Hammel et al., "Mechanism of efficient double-strand break repair by a long non-coding RNA," Nucleic Acids Research, vol. 48, no. 19, pp. 10953-10972, 2020. 
[20] H. Cho, Y. Li, S. Archacki et al., "Splice variants of lncRNA RNA ANRIL exert opposing effects on endothelial cell activities associated with coronary artery disease," RNA Biology, vol. 17, no. 10, pp. 1391-1401, 2020.

[21] Y. C. Feng, X. Y. Liu, L. Teng et al., "c-Myc inactivation of p53 through the pan-cancer lncRNA MILIP drives cancer pathogenesis," Nature Communications, vol. 11, no. 1, p. 4980, 2020.

[22] S.-X. Liu, F. Zheng, K.-L. Xie, M.-R. Xie, L.-J. Jiang, and Y. Cai, "Exercise reduces insulin resistance in type 2 diabetes mellitus via mediating the lncRNA MALAT1/MicroRNA382-3p/Resistin axis," Molecular Therapy-Nucleic Acids, vol. 18, pp. 34-44, 2019.

[23] D. L. Chen, D. Y. Shen, C. K. Han, and Y. Tian, "LncRNA MEG3 aggravates palmitate-induced insulin resistance by regulating miR-185-5p/Egr2 axis in hepatic cells," European Review for Medical and Pharmacological Sciences, vol. 23, pp. 5456-5467, 2019.

[24] B. Li, Y. Zhou, J. Chen et al., "Long noncoding RNA H19 acts as a miR-29b sponge to promote wound healing in diabetic foot ulcer," The FASEB Journal, vol. 35, no. 1, Article ID e20526, 2021.

[25] Y. Wang, Y. Hu, C. Sun et al., "Down-regulation of Risa improves insulin sensitivity by enhancing autophagy," The FASEB Journal, vol. 30, no. 9, pp. 3133-3145, 2016.

[26] X. Shen, Y. Zhang, X. Zhang et al., "Long non-coding RNA Bhmt-AS attenuates hepatic gluconeogenesis via modulation of Bhmt expression," Biochemical and Biophysical Research Communications, vol. 516, no. 1, pp. 215-221, 2019.

[27] L. Shu, G. Hou, H. Zhao, W. Huang, G. Song, and H. Ma, "Resveratrol improves high-fat diet-induced insulin resistance in mice by downregulating the lncRNA NONMMUT008655.2," American Journal of Translational Research, vol. 12, no. 1, pp. 1-18, 2020.

[28] L. Shu, G. Hou, H. Zhao, W. Huang, G. Song, and H. Ma, "Long non-coding RNA expression profiling following treatment with resveratrol to improve insulin resistance," Molecular Medicine Reports, vol. 22, no. 2, pp. 1303-1316, 2020.

[29] Z. Liu, Z. Zhang, G. Song, X. Wang, H. Xing, and C. Wang, "Resveratrol improves skeletal muscle insulin resistance through downregulating lncRNA NONMMUT044897.2," Research Square, 2021.

[30] K. J. Livak and T. D. Schmittgen, "Analysis of relative gene expression data using real-time quantitative PCR and the $2^{-\Delta \Delta \mathrm{CT}}$ method," Methods, vol. 25, no. 4, pp. 402-408, 2001.

[31] M. Liu, J. Qin, Y. Hao et al., "Astragalus polysaccharide suppresses skeletal muscle myostatin expression in diabetes: involvement of ROS-ERK and NF- $\kappa \mathrm{B}$ pathways," Oxidative Medicine and Cellular Longevity, vol. 2013, Article ID 782497 , 10 pages, 2013.

[32] E. Abbasi Oshaghi, M. T. Goodarzi, V. Higgins, and K. Adeli, "Role of resveratrol in the management of insulin resistance and related conditions: mechanism of action," Critical Reviews in Clinical Laboratory Sciences, vol. 54, no. 4, pp. 267-293, 2017.

[33] A. Sadeghi, S. S. Seyyed Ebrahimi, A. Golestani, and R. Meshkani, "Resveratrol ameliorates palmitate-induced inflammation in skeletal muscle cells by attenuating oxidative stress and JNK/NF- $\kappa$ B pathway in a SIRT1-independent mechanism," Journal of Cellular Biochemistry, vol. 118, no. 9, pp. 2654-2663, 2017.

[34] L. Lu, X. Ye, Q. Yao et al., "Egr2 enhances insulin resistance via JAK2/STAT3/SOCS-1 pathway in HepG2 cells treated with palmitate," General and Comparative Endocrinology, vol. 260, pp. 25-31, 2018.

[35] X. Huang, G. Liu, J. Guo, and Z. Su, "The PI3K/AKT pathway in obesity and type 2 diabetes," International Journal of Biological Sciences, vol. 14, no. 11, pp. 1483-1496, 2018.

[36] A. M. Cardoso, C. M. Morais, O. Rebelo et al., "Downregulation of long non-protein coding RNA MVIH impairs glioblastoma cell proliferation and invasion through an miR302a-dependent mechanism," Human Molecular Genetics, vol. 30, no. 1, pp. 46-64, 2021.

[37] J. Zheng, Q. Tan, H. Chen et al., "IncRNA-SNHG7-003 inhibits the proliferation, migration and invasion of vascular smooth muscle cells by targeting the miR-1306-5p/SIRT7 signaling pathway," International Journal of Molecular Medicine, vol. 47, no. 2, pp. 741-750, 2021. 